\title{
The multifaceted role of ferroptosis in liver disease
}

\author{
Junyi Chen $\mathbb{D}^{1,2,3}$, Xiaopeng $\mathrm{Li} \mathbb{D}^{1,2,3}$, Chaodong Ge $\mathbb{D}^{1,3}$, Junxia Min $\mathbb{D}^{1 凶}$ and Fudi Wang $\mathbb{D}^{1,2 \bowtie}$
}

(c) The Author(s) 2022

Ferroptosis is an iron-dependent form of non-apoptotic cell death characterized by excessive lipid peroxidation and associated with a plethora of pathological conditions in the liver. Emerging evidence supports the notion that dysregulated metabolic pathways and impaired iron homeostasis play a role in the progression of liver disease via ferroptosis. Although the molecular mechanisms by which ferroptosis causes disease are poorly understood, several ferroptosis-associated genes and pathways have been implicated in liver disease. Here, we review the physiological role of the liver in processing nutrients, our current understanding of iron metabolism, the characteristics of ferroptosis, and the mechanisms that regulate ferroptosis. In addition, we summarize the role of ferroptosis in the pathogenesis of liver disease, including liver injury, non-alcoholic steatohepatitis, liver fibrosis, liver cirrhosis, and hepatocellular carcinoma. Finally, we discuss the therapeutic potential of targeting ferroptosis for managing liver disease.

Cell Death \& Differentiation (2022) 29:467-480; https://doi.org/10.1038/s41418-022-00941-0

\section{FACTS}

- Liver disease is on the rise and now accounts for approximately two million deaths annually worldwide.

- Several forms of programmed cell death, including apoptosis, necroptosis, and ferroptosis, have been implicated in the pathogenesis of various liver diseases. With respect to liver injury, steatohepatitis, fibrosis, and cirrhosis, liver cells are more susceptible to ferroptosis; in contrast, in liver cancer the cancer cells are either intrinsically resistant to ferroptosis or acquire resistance to ferroptosis.

- A growing body of evidence suggests that ferroptosis may serve as a promising target for the prevention and treatment of many forms of liver disease.

\section{OPEN QUESTIONS}

- What physiological role, if any, does ferroptosis play in the liver?

- Can we identify reliable, sensitive biomarkers of ferroptosis in liver disease?

- When should we target ferroptosis in specific pathological liver conditions and/or disease stages?

- In treating liver cancer, can we activate ferroptosis specifically in cancer cells without affecting healthy cells?

\section{INTRODUCTION}

Liver disease is a major cause of death worldwide [1], and cell death plays a key role in driving the progression of various forms of liver disease. With respect to regulated cell death, emerging evidence indicates that both necroptosis and ferroptosis play important roles in the pathogenesis of liver disease [2-5]. The pathophysiological relevance of ferroptosis was first demonstrated in vivo in ischemia/reperfusion injury (IRI) both in the liver and the kidneys [4]; since then, numerous studies have shown that lipid peroxidation and ferroptosis play a central role in a plethora of liver disease models. Moreover, blocking hepatic cell death can provide a cost-effective strategy for protecting the liver against injury and related diseases.

Ferroptosis is an iron-dependent form of cell death accompanied by large levels of lipid peroxidation and is distinct from other types of cell death such as apoptosis, autophagy, and pyroptosis [6, 7]. In addition to the role of iron metabolism in ferroptosis, an increasing number of other metabolic pathways have been implicated in ferroptosis, including the cyst(e)ine/ glutathione $(\mathrm{GSH}) /$ glutathione peroxidase 4 (GPX4) axis, the guanosine triphosphate cyclohydrolase $1(\mathrm{GCH} 1) /$ tetrahydrobiopterin $\left(\mathrm{BH}_{4}\right) /$ dihydrofolate reductase (DHFR) axis, and the ferroptosis suppressor protein 1 (FSP1)/ coenzyme Q (CoQ) axis [8]. A growing number of studies suggest that ferroptosis plays an important role in the pathogenesis of various types of liver disease, including hemochromatosis, alcohol-associated liver disease (ALD), hepatitis C virus (HCV) infection, non-alcoholic steatohepatitis (NASH), and hepatocellular carcinoma (HCC) $[9,10]$. Thus, targeting ferroptosis may provide a promising new therapeutic strategy for treating patients with liver disease.

Here, we review current knowledge regarding the role of ferroptosis in various forms of liver disease, and we provide new perspectives regarding the physiological role of the liver in processing nutrients and iron metabolism, as well as the potential

\footnotetext{
'The First Affiliated Hospital, The Fourth Affiliated Hospital, Institute of Translational Medicine, School of Public Health, Cancer Center, State Key Laboratory of Experimental Hematology, Zhejiang University School of Medicine, 310058 Hangzhou, China. ${ }^{2}$ The First Affiliated Hospital, The Second Affiliated Hospital, Basic Medical Sciences, School of Public Health, Hengyang Medical School, University of South China, 421001 Hengyang, China. ${ }^{3}$ These authors contributed equally: Junyi Chen, Xiaopeng Li, Chaodong Ge.

凶email: junxiamin@zju.edu.cn; fwang@zju.edu.cn

Edited by E. Baehrecke
}

Received: 22 November 2021 Revised: 12 January 2022 Accepted: 13 January 2022

Published online: 24 January 2022 


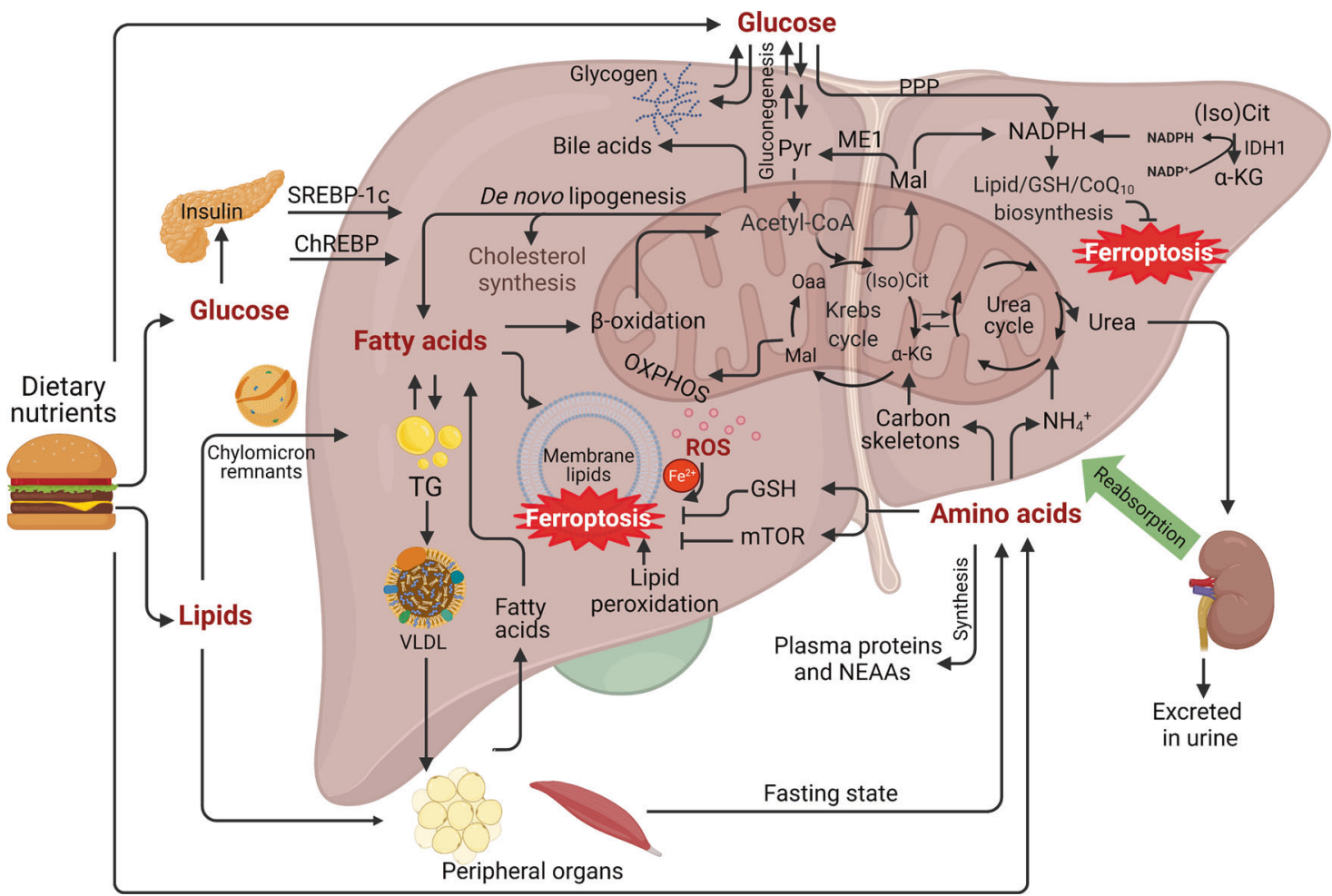

Fig. 1 Hepatic glucose, lipids, and amino acid metabolism. The liver is an essential organ for controlling many physiological metabolic processes, including the metabolism of glucose, lipids, and amino acids. After a meal, glucose rapidly enters the liver and is sequestered as glycogen. Glucose produces energy via glycolysis and generates NADPH the PPP pathway. Two other major pathways also participate in the generation of NADPH by ME1 and IDH1. In addition, excess glucose stimulates de novo lipogenesis in the liver. During fasting, the liver secretes glucose both by breaking down glycogen (glycogenolysis) and by de novo glucose synthesis (gluconeogenesis). With respect to lipid metabolism, glycolytic products are used to synthesize fatty acids through de novo lipogenesis. The majority of chylomicrons are delivered to the adipose tissue and skeletal muscle, while chylomicron remnants are cleared by the liver. VLDL cholesterol is also assembled in the liver from triglycerides, cholesterol, and apolipoproteins, and used to transport endogenous TG to other peripheral organs. The liver is also the site of deamination, amino acid transamination, and urea synthesis. SLC7A11-mediated cystine uptake is essential for GSH synthesis, which inhibits ferroptosis. As a sensor of amino acids, mTORC1 activation can suppress ferroptosis by increasing GPX4 levels. Finally, altered glucose, lipid, and amino acid metabolism can lead to insulin resistance, NAFLD, and cholestasis. $\alpha$-KG, $\alpha$-ketoglutarate; ChREBP, carbohydrateresponsive element-binding protein; GSH, glutathione; IDH1, isocitrate dehydrogenase 1; Mal, malate; (Iso) Cit, (Iso)citrate; ME1, malic enzyme 1; mTOR, mechanistic target of rapamycin; NADPH, nicotinamide adenine dinucleotide phosphate; NAFLD, non-alcoholic fatty liver disease; NEAAs, non-essential amino acids; Oaa, oxaloacetate; OXPHOS, oxidative phosphorylation; PPP, pentose phosphate pathway; Pyr, pyruvate; ROS, reactive oxygen species; SREBP1c, sterol regulatory element-binding transcription factor 1c; TG, triglyceride; VLDL, very low-density lipoprotein.

mechanisms that underlie hepatic cell death and the pathophysiological pathways involved in liver disease.

\section{Hepatic metabolism of glucose, lipids, amino acids, and ferroptosis}

The liver functions as the body's metabolic hub for nutrients such as glucose, lipids, and amino acids (Fig. 1). Dysregulation of these macronutrients can lead to oxidative stress, liver disease, and even mortality [11, 12]. An increasing number of hepatic metabolic pathways have been associated with ferroptosis [13, 14]. For instance, NADPH levels decrease significantly upon induction of ferroptosis [14]. The metabolic NADPH pathway protects against ferroptosis by mediating the synthesis of $\mathrm{GSH}$ and $\mathrm{CoQ}_{10}$; thus, NADPH has been used as a biomarker for ferroptosis [15-18].

The metabolism of essential fatty acids (EFAs) is particularly important, as the accumulation of lipid peroxidation is considered one of the major characteristics of ferroptosis [19]. Non-alcoholic fatty liver disease (NAFLD) is characterized by abnormal lipid deposition in the liver. Importantly, prolonged overnutrition can increase the progression of NAFLD to NASH with fibrosis $[20,21]$.

Some amino acids are linked directly to ferroptosis by regulating oxidative stress. For example, cysteine is the limiting amino acid in GSH synthesis, and inhibiting its import through the cystine/glutamate antiporter SLC7A11 is sufficient to induce ferroptosis by depleting GSH levels [22]. Importantly, cystine deprivation has been shown to induce ferroptosis in a wide range of cancer cell lines. However, Conlon et al. [23] recently reported that cystine deprivation failed to induce ferroptosis when other amino acids such as arginine are also absent, suggesting that amino acids play a complex role in mediating the cellular response to ferroptosis inhibitors.

\section{Both systemic and hepatic iron hemostasis are tightly regulated}

Systemic iron homeostasis is tightly regulated in order to ensure that the body contains sufficient iron due to dietary iron absorption and to prevent the toxic accumulation of iron (Fig. 2). In addition, iron metabolism is also regulated primarily by the liver, which maintains systemic iron balance by producing and secreting hepcidin, the master regulator of iron homeostasis [24] (Fig. 2). Hepatocytes acquire transferrin-bound iron (TBI) and nontransferrin-bound iron (NTBI) via TFR1 and SLC39A14, respectively [25-27]. In hepatocytes, iron is stored in ferritin and exported by ferroportin (FPN) [28] (Fig. 3). Mon1a was identified as a modifier gene of cellular iron metabolism via trafficking of FPN to the surface of iron-recycling macrophages [29]. Hepcidin inhibits 


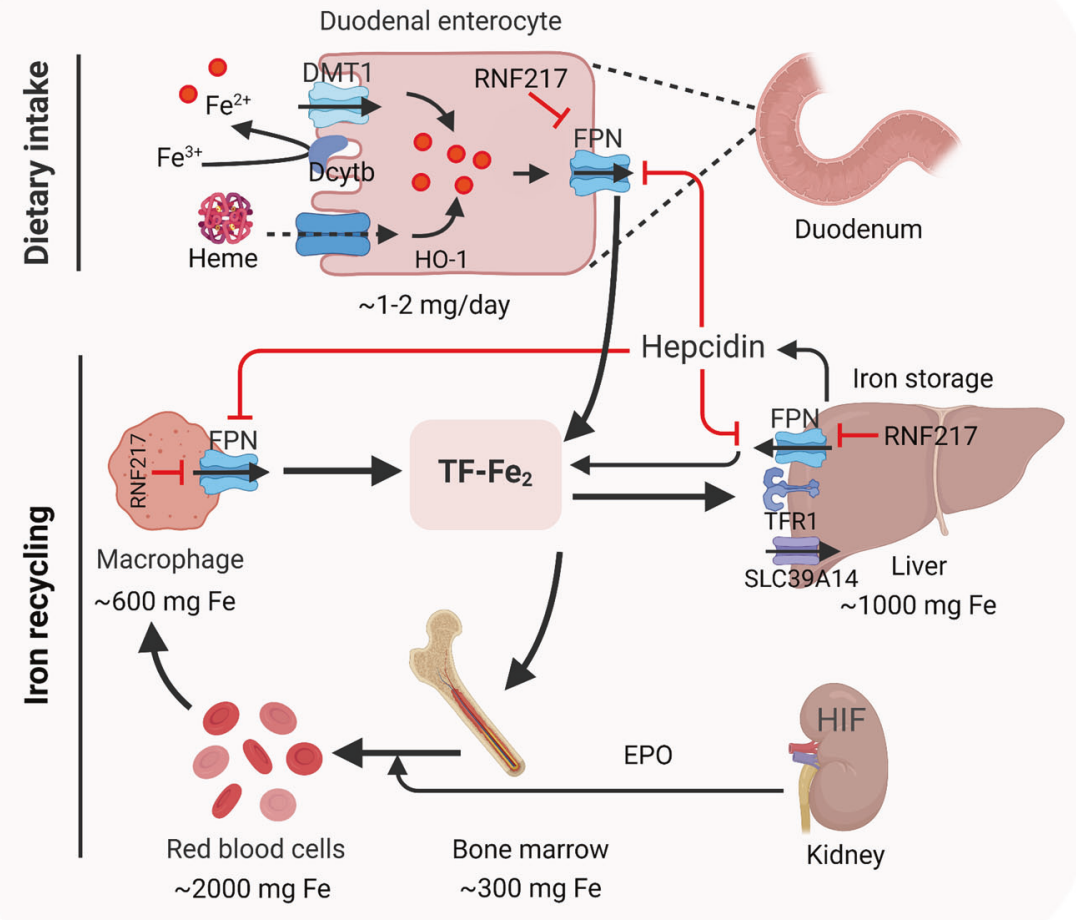

Fig. 2 Regulation of systemic iron homeostasis. After intake of iron, $\mathrm{Fe}^{3+}$ is reduced by dcytb and then transported into enterocyte through DMT1. Dietary heme is absorpbed by unknown mechanism and degraded in enterocyte by HO-1. Once exported by FPN, Fe ${ }^{3+}$ binds to transferrin (diferric transferrin, TF-Fe 2 ), travels to tissues, and largely utilized in new red blood cells. Macrophage degraded senescent RBCs to recycle iron. Once needed, EPO, released by kidney, promotes erythropoiesis by HIF signaling pathway. The iron utilization of erythroid marrow and its recycling by macrophages represent the major iron circulation. Excess iron can be stored in hepatocytes through TFR1mediated TF-Fe $e_{2}$ or SLC39A14-participated non-transferrin-bound iron (NTBI). The release of iron from enterocyte, red blood cells, and macrophages is precisely controlled by FPN, the body's sole iron exporter, to maintain a relatively stable iron level. The peptide hepcidin, the master regulator of systemic iron homeostasis, is a circulating hormone synthesized by the liver. Recently, we identified RNF217 as a novel E3 ligase for mediating FPN degradation. Dcytb, duodenal cytochrome b; DMT1, divalent metal transporter 1; EPO, erythropoietin; FPN, ferroportin; TFR1, transferrin receptor 1; HO-1, heme oxygenase 1; HIF, hypoxia induced factor; RBCs, red blood cells; NTBI, non-transferrinbound iron.

cellular iron efflux by binding to and inducing the degradation of FPN. RNF217 was identified as a novel E3 ubiquitin ligase and was shown to play a role in mediating the degradation of FPN [30]. Notably, we previously showed that iron overload can directly induce ferroptosis in a variety of organs, including the liver and heart $[14,26,31]$.

\section{Dysregulated iron metabolism triggers ferroptosis}

Lipid peroxidation and toxic ROS result from the iron-mediated Fenton reaction and enzymatic oxygenation, which are hallmark features of ferroptosis [32]. Impaired iron homeostasis plays a role in the pathogenesis of various diseases by triggering ferroptosis, with recent reports implicating transferrin and its cell surface TFR1 as key regulators of ferroptosis [33]. Interestingly, CISD1 (CDGSH iron-sulfur domain-containing protein 1, also known as MitoNEET) inhibits ferroptosis by altering the accumulation of mitochondrial iron [34]. We recently showed that hepatic SLC39A14 accelerates iron-induced ferroptosis in the liver of hepatic Trf knockout mice via its ability to transport NTBI, revealing a novel molecular mechanism by which ferroptosis drives the pathogenesis of liver fibrosis and cirrhosis [26].

Increased iron uptake and reduced iron storage may lead to iron overload, subsequently triggering ferroptosis. This hypothesis was tested using iron chelators and iron supplements during erastin-mediated ferroptosis [17]. Nuclear receptor coactivator 4 (NCOA4)-mediated ferritinophagy, a specific form of autophagy, has been shown to induce ferroptosis by degrading ferritin and inducing iron overload [35]. The iron chaperone activity of PCBP1 is required for preventing iron-mediated toxicity and preventing ferroptosis [36]. Taken together, these studies indicate that iron metabolism is tightly regulated at multiple levels through the ferroptosis pathway (Fig. 4).

\section{Accumulation of lipid peroxides drives ferroptosis}

Polyunsaturated fatty acids (PUFAs) containing bis-allylic carbons are highly susceptible to lipid peroxidation [37], and recent findings suggest that a wide range of PUFAs may be involved in the ferroptosis pathway [15]. Inhibiting related enzymes such as fatty acid desaturase 2 (FADS2) and acetyl-CoA carboxylase (ACC) confers resistance to lipid peroxidation and ferroptosis induced by the compound RSL3 $[38,39]$. Recently, a genetic screen revealed two lipid metabolism regulators-lysophosphatidylcholine acyltransferase 3 (LPCAT3) and acyl-CoA synthetase long-chain family member 4 (ACSL4) - that drive ferroptosis via an accumulation of oxidized phospholipids in the cell membrane [40]. ACSL4 preferentially catalyzes the esterification of PUFAs, including AA, with CoA. PUFA-CoAs are subsequently used for the synthesis of phospholipids, including phosphatidylethanolamine, and loss of ACSL4 results in significant resistance to ferroptosis induced by GPX4 deficiency [41]; in contrast, knocking out LPCAT3 does not confer robust protection against ferroptosis, suggesting that these family members may have compensatory mechanisms [41]. It is important to note that "lipid peroxidation" is an ambiguous concept, as its products can form spontaneously by 


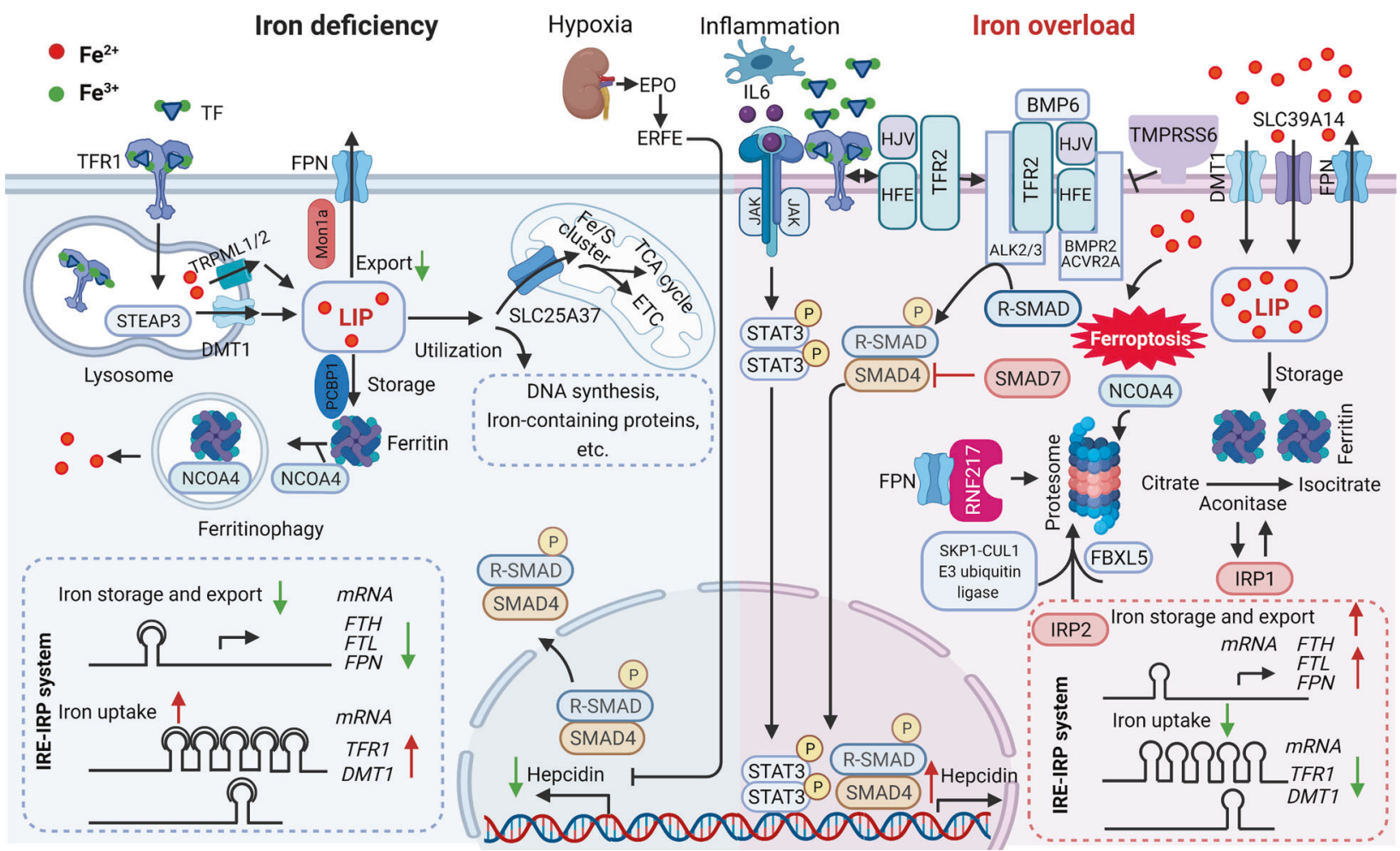

Fig. 3 Iron metabolism in the liver. Under iron-deficient conditions (left), the majority of iron is bound to transferrin (TF), which binds to the transferrin receptor 1 (TFR1) at the cell surface followed by receptor-mediated endocytosis, resulting in ferric iron being released from TF and reduction to ferrous iron by an lysosomal reductase such as STEAP3. The ferrous iron is then transported into the lysosomal membrane by DMT1 and TRPML1/2, where it becomes part of the labile iron pool in the cytosol. Labile iron can be stored in the iron-storage protein ferritin or used to synthesize heme and iron-sulfur clusters in the mitochondria or in the cytosol. Iron can also be exported from the cell by the body's sole iron exporter, ferroportin (FPN). In addition, the IRE/IRP system regulates the expression of iron-related proteins such as TFR1, ferritin and FPN, upregulating TFR1 and DMT1 expression and downregulating FPN and FTH/FTL expression. During iron overload (right), hepcidin expression is upregulated by either the canonical bone morphogenetic protein (BMP)/SMAD pathway or by IL-6-pSTAT3 inflammatory signaling, which in turn limits iron absorption by increasing FPN degradation. In response to excess iron, BMP6, together with HJV, activates type 1 (Alk2/3)and type 2 (BMPR2, ACVR2A) BMP serine threonine kinase receptors to phosphorylate R-SMAD (receptor-activated SMAD), leading to activation of BMP/SMAD signaling pathway. High concentration of TF-Fe ${ }_{2}$ interact with TFR1, resulting in forming complex of TFR2/HJV/HFE to enhance the BMP/SMAD signaling in regulating hepcidin. TMPRSS6 inhibits BMP/SMAD signaling by cleaving HJV. The IRP system not only downregulates iron uptake-related genes such as TFR1 and DMT1 expression, it also upregulates FPN and FTH/FTL expression. IRP2 mediated by SKP1-CUL1 E3 ubiquitin ligase and NCOA4 are degraded, while IPR1 works as aconitase to convert citrate to isocitrate due to conformational change. RNF217 is a recently identified E3 ligase that regulates the degradation of FPN. Non-transferrin-bound iron (NTBI) enters hepatocytes through metal transporter proteins such as SLC39A14, and the increased iron can directly induce ferroptosis. ACVR2A, activin receptor type-2A; ALK, activin receptor-like kinase; BMP6, bone morphogenetic protein 6; BMPR2, bone morphogenetic protein receptor type 2; DMT1, divalent metal transporter 1; EPO, erythropoietin; ERFE, erythroferrone; ETC, electron transport chain; FBXL5, F-box/LRR-repeat protein 5; FPN, ferroportin; FTH, ferritin heavy chain; FTL, ferritin light chain; JAK, Janus kinase; LIP, labile iron pool; NCOA4, nuclear receptor coactivator 4; NTBI, non-transferrinbound iron; HJV, hemojuvelin; IL-6, interleukin 6; IRE, iron-responsive elements; IRP, iron-regulatory proteins; SLC39A14, solute carrier family 39 member 14; SMAD4, SMAD family member 4; SMAD7, SMAD family member 7; STAT3, signal transducer and activator of transcription 3; STEAP3, six-transmembrane epithelial antigen of prostate 3; TCA cycle, tricarboxylic acid cycle; TFR1, transferrin receptor 1; TFR2, transferrin receptor 2; TMPRSS6, transmembrane protease serine 6; TRPML1/2, Mucolipin TRP channel 1/2;UTRs, untranslated regions.

either autoxidation (mediated by carbon- and oxygen-centered radicals) or enzyme-catalyzed processes $[42,43]$. In addition to the non-specific propagation of radicals, lipid peroxidation can also be accelerated by cyclooxygenases (COXs) [44], cytochrome p450 oxidoreductase (POR), and lipoxygenases (LOXs) via a controlled process $[42,45]$.

Whether a cell undergoes ferroptosis is largely determined by intertwined signals from subcellular organelles such as mitochondria, lysosomes, and peroxisomes, which coordinately regulate ROS production as well as lipid oxidation in the process of ferroptosis [46]. Emerging evidence supports the notion that mitochondria are an important regulator of ferroptosis through the integrative regulation of ROS production, the TCA cycle, iron metabolism, and mitochondrial DNA [47]. Lysosomes participate in ferroptosis primarily through the activation of selective autophagy processes (e.g., ferritinophagy and lipophagy) and by lysosomal accumulation of iron and nitric oxide [35, 48]. Using CRISPR screening and lipidomics profiling, Zou et al. [45] recently found that peroxisomes induce ferroptosis by synthesizing polyunsaturated ether phospholipids (PUFA-ePLs), which are substrates of lipid peroxidation. Although significant advances have been made toward unraveling the signaling pathways involved in the production of lipid peroxidation, the specific contribution of various organelles in modulating ferroptosis remains an open question.

\section{The GSH/GPX4 axis inhibits ferroptosis}

The GSH/GPX4 axis plays an essential role in counteracting the production of specific phospholipid hydroperoxides in the presence of catalytically active iron. The upstream component in this system is system $\mathrm{x}_{\mathrm{c}}{ }^{-}$, a cystine-glutamate antiporter composed of the transporter protein SLC7A11 linked via a 


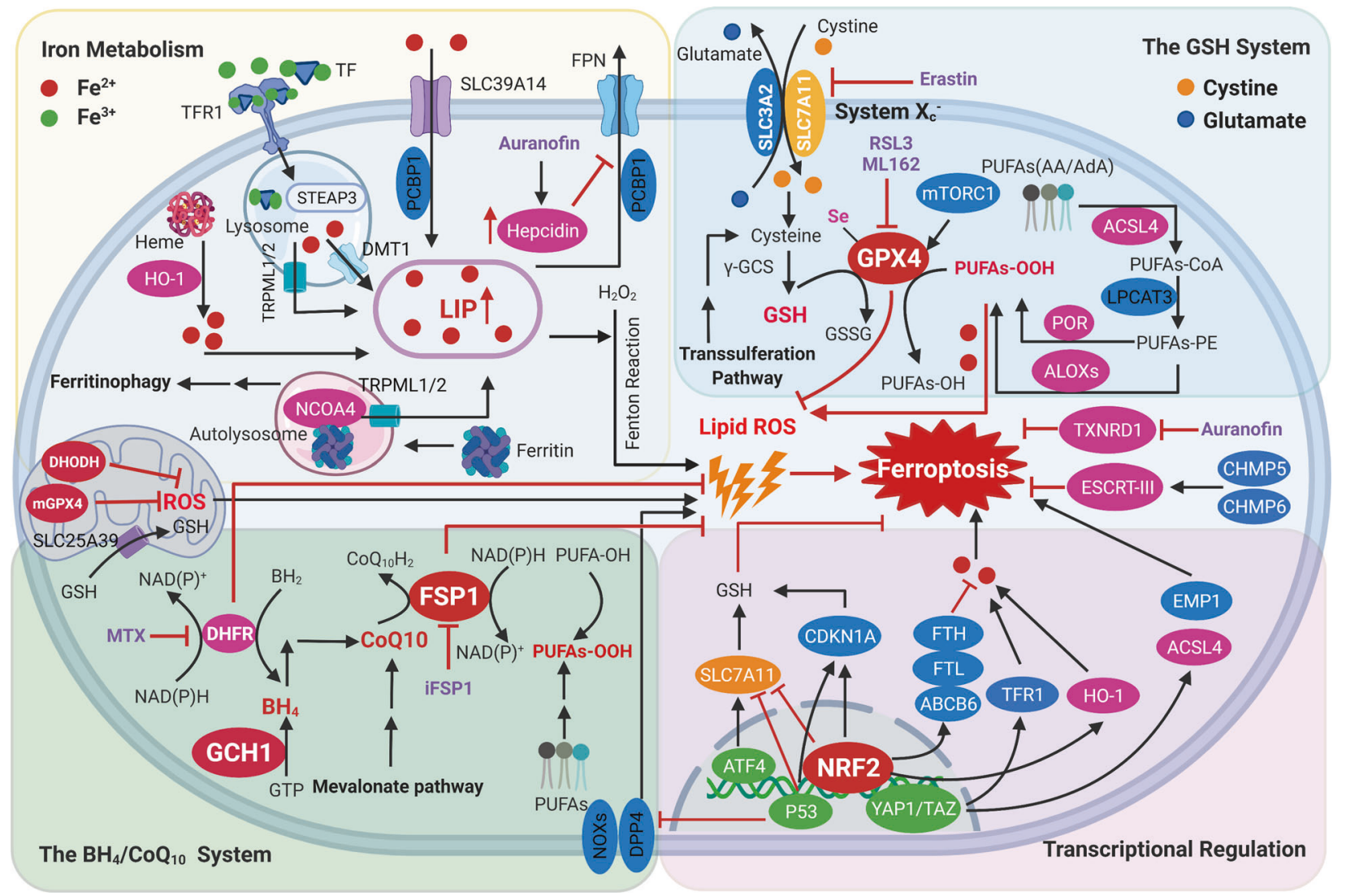

Fig. 4 Regulatory signaling pathways and defense mechanisms implicated in the process of ferroptosis. Iron metabolism has been associated with ferroptosis (top left). As the body's principal organ for storing excess iron and synthesizing hepcidin, the liver serves as the functional cornerstone in maintaining iron homeostasis. When cellular iron is sufficient, transferrin-bound iron decreases in order to limit excessive iron accumulation. Under iron-overload conditions, excess iron results in redox-active non-transferrin-bound iron (NTBI), the uptake of which is mediated by metal transporter proteins such as SLC39A14. In general, excess iron is stored in ferritin and free iron existing as part of the LIP can be accumulated due to imbalanced metabolism. NOCA4 has been shown to act as a cargo receptor that binds to ferritin delivering it to the autolysosome, resulting in releasing of free iron. Free iron participates in the generation of ROS through Fenton reaction. ACSL4 and LPCAT3 are necessary for ferroptosis to produce PUFAs-PE. The activation of ALOXs, POR, or NOXs promotes lipid peroxidation, yet the activation of ESCRT complex repairs cell membrane damage. Lipid peroxidation of membrane phospholipids can be eliminated by three parallel metabolic pathways, including the cyst(e)ine/GSH/GPX4 axis (top right), as well as the CoQ 10 /FSP1 and GCH1/BH4/DHFR axis (bottom left). In mitochondrial, DHODH and mitoGPX4 participated in ROS scavenging. In addition, several transcription factors such as p53, NRF2, ATF4, and YAP/TAZ regulate ferroptosis-related genes upon oxidative stress (bottom right). ABCB6, ATP-binding cassette subfamily B member 6; LOXs, lipoxygenases; ACSL4, acyl-CoA synthetase long-chain family member 4; ATF4, activating transcription factor 4; BACH1, BTB domain and $\mathrm{CNC}$ homolog 1; $\mathrm{BH}_{2}$, 7,8-dihydrobiopterin; $\mathrm{BH}_{4}$, tetrahydrobiopterin; CDKN1A, cyclin-dependent kinase inhibitor p21; $\mathrm{CHMP5/6}$, chromatin modeling protein 5/6; $\mathrm{CoQ}_{10} \mathrm{H}_{2}$, ubiquinol; $\mathrm{GCH} 1$, guanosine triphosphate cyclohydrolase 1; DHFR, dihydrofolate reductase; $\mathrm{DHODH}$, dihydroorotate dehydrogenase; DDP4, dipeptidyl peptidase-4; DMT1, divalent metal transporter1; EMP1, epithelial membrane protein 1; ESCRT-III, endosomal sorting complex required for transport III; FPN, Ferroportin; FSP1, ferroptosis suppressor protein 1; FTH, ferritin heavy chain; FTL, ferritin light chain; GCS, glutamylcysteine synthetase; GPX4, glutathione peroxidase 4; GCH1, guanosine triphosphate cyclohydrolase 1; GSH, glutathione; HO-1, heme oxygenase 1; LIP, labile iron pool; LPCAT3, lysophosphatidylcholine acyltransferase 3; MTX, methotrexate; mTORC1, mechanistic target of rapamycin complex 1; NRF2, nuclear factor erythroid 2-related factor 2; NCOA4, nuclear receptor coactivator 4; PCBP, poly (RC)-binding proteins; POR, NADPH-cytochrome P450 reductase; PUFA, polyunsaturated fatty acid; ROS, reactive oxygen species; STEAP3, six-transmembrane epithelial antigen of prostate 3; TF, transferrin; TFR1, transferrin receptor 1; TXNRD1, thioredoxin reductase 1; TRPML1/2, Mucolipin TRP channel 1/2.

disulfide bond to the regulatory subunit SLC3A2. Inhibition of system $\mathrm{x}_{\mathrm{c}}{ }^{-}$activates cellular ferroptosis in cell lines due to a lack of intracellular cysteine. The transsulfuration pathway, which transfers sulfur from homocysteine to cysteine, regulates ferroptosis in cells in which system $x_{c}{ }^{-}$is inhibited [49]. Recent studies found that transcription factors such as p53, the tumor suppressor BAP1 (BRCA1-associated protein 1), and ATF3 (activating transcription factor 3 ) increase cellular susceptibility to ferroptosis via decreased cystine uptake by inhibiting SLC7A11 expression [50-52]. Interestingly, OTUB1 (OTU domain-containing ubiquitin aldehyde-binding protein 1) has also been shown to play a key role in SLC7A11 stability and sensitivity to ferroptosis [53].
GSH is generated by de novo synthesis in a process mediated by two ATP-dependent ligases (glutamate-cysteine ligase and glutathione synthase), as well as by the regeneration of GSH from GSSG, a process catalyzed by glutathione disulfide reductase. Endogenous cysteine is generated primarily via GSH or the TXNRD1 (thioredoxin reductase 1)-mediated reduction of cystine [54]. High doses of the anti-rheumatic drug auranofin have been shown to activate ferroptosis by inhibiting TXNRD1 activity [55].

Finally, GPX4 is a well-characterized core suppressor of ferroptosis that directly reduces lipid hydroperoxide to form non-toxic lipid alcohol. GSH serves as a cofactor for the selenoenzyme GPX4, and inhibiting the de novo synthesis of GSH synthesis induces ferroptosis by inactivating GPX4 [56]. GPX4 
also plays a role in the development and maintenance of a variety of physiological functions, as global knockout of Gpx4 in mice causes embryonic lethality, whereas conditional Gpx4 knockout mice develop disorders in the endothelium, liver, brain, kidney, immune system, and hematopoietic system [57]. As a selenocysteine-containing enzyme, GPX4 has mitochondrial, cytosolic, and nuclear isoforms, all of which are encoded by the same gene. Mitochondrial or nuclear Gpx4 deletion in mice causes male infertility without impairing embryogenesis or postnatal development $[58,59]$. Together, these in vivo animal studies suggest that mitochondrial and/or nuclear GPX4 plays an important role in mammalian male fertility, while cytosolic GPX4 is essential for embryonic development and cell survival.

\section{The NAD(P)H/FSP1/CoQ 10 system suppresses ferroptosis}

To identify GPX4-independent ferroptosis-resistance genes, two groups independently performed a synthetic lethal CRISPR-Cas9 screen [15] and a gain-of-function screen [16]. Interestingly, both studies identified FSP1 (encoding ferroptosis suppressor protein 1) as a novel ferroptosis-resistance gene $[15,16]$. FSP1's antiferroptotic function is independent of cellular GSH levels, GPX4 activity, ACSL4 expression, and oxidizable FA content, suggesting a new anti-ferroptosis mechanism [15, 16]. Overexpressing FSP1 in cells lacking GPX4 expression was shown to significantly reduce specific phospholipid peroxidation products compared to control cells $[15,16]$. Moreover, cells lacking FSP1 expression have increased sensitivity to ferroptosis inducers, including the GPX4 inhibitor ML162 and the system $x_{c}^{-}$inhibitor erastin $[15,16]$. Recently, Dai et al. [51] found that FSP1 prevents ferroptosis by promoting ESCRT-III (endosomal sorting complex required for transport III)-mediated membrane repair in a $\mathrm{CoQ}_{10}$-independent manner. Based on currently available evidence, both FSP1-CoQ 10 and ESCRT-III play a role in repairing membrane damage during ferroptosis. Given that $\mathrm{CoQ}_{10}$ is an essential cofactor in the mitochondrial electron transport pathway, as well as a lipidsoluble antioxidant, it is reasonable to speculate that FSP1-CoQ 10 and ESCRT-III have complementary functions. Taken together, these findings indicate that FSP1 blocks ferroptosis via a complex interplay between regulatory pathways.

\section{The $\mathrm{GCH} 1 / \mathrm{BH}_{4} / \mathrm{DHFR}$ system suppresses ferroptosis}

The metabolic change that occurs in cancer cells often requires endogenous antioxidants to reduce toxic lipid oxidation. Both the cyst(e)ine/GSH/GPX4 system and the NAD(P)H/FSP1/CoQ ${ }_{10}$ system have been implicated in the metabolic processes related to the cellular response to lipid peroxidation [15-17] (Fig. 4). Genomewide CRISPR activation screening identified guanosine triphosphate cyclohydrolase $1(\mathrm{GCH} 1)$, the rate-limiting enzyme in the synthesis of $\mathrm{BH}_{4}$, as a potent GPX4-independent ferroptosisinhibiting factor. $[60,61]$. The $\mathrm{BH}_{4}$ molecule serves as an essential cofactor for a number of enzymes involved in central metabolic processes, including tryptophan hydroxylases, nitric oxide synthase (NOS), alkylglycerol monooxygenase, and isoenzymes. Importantly, $\mathrm{BH}_{4}$ is used not only as a direct antioxidant to protect cells from lipid peroxidation and ferroptosis, but also in the synthesis of $\mathrm{CoQ}_{10}[60,61]$. Nevertheless, further studies are needed in order to elucidate the antioxidant mechanisms involving the $\mathrm{GCH} 1 / \mathrm{BH}_{4} / \mathrm{DHFR}$ system in the context of ferroptosis.

\section{Mitochondrial GPX4 and DHODH suppress ferroptosis}

Mitochondria are essential for maintaining cellular viability, as they perform critical functions in bioenergetics, metabolism, and various signaling pathways. The primary function of mitochondria is to generate ATP via oxidative phosphorylation using the electron transport chain. It has long been recognized that ROS are produced during mitochondrial respiration and that mitochondria serve as the cell's principal source of ROS production.
ROS trigger lipid peroxidation-induced cell death by reacting with PUFAs in the lipid bilayers. Moreover, a number of molecular, cellular, pharmacological, and metabolomics studies indicate that mitochondrial metabolic activity is required for generating sufficient levels of ROS to trigger ferroptosis [47, 62]. In response to various forms of oxidative damage, cells use multiple protective and/or repair systems to reduce the effects of toxic modified membrane lipids. To prevent damage due to lipid peroxides, mammalian cells rely primarily on three systems involving GPX4, FSP1, and DHFR. Recently, however, Mao et al. [63] found that the enzyme dihydroorotate dehydrogenase (DHODH) and mitochondrial GPX4 constitute two additional defense enzymes that can detoxify lipid peroxides in the mitochondria. As an iron-containing flavin-dependent enzyme, DHODH couples the pyrimidine biosynthesis pathway to the mitochondrial respiratory chain, suggesting possible strategies for treating $\mathrm{GPX} 4^{\text {low }}$ cancers by targeting DHODH [63].

\section{Oxidative stress-related transcription factors regulate ferroptosis}

Ferroptosis is a highly complex and modifiable process that requires transcription factors to either repress or activate the expression of ferroptosis-related genes. The functions of various transcription factors such as TP53, NRF2, ATF4, YAP1, and TAZ in ferroptosis are summarized in Fig. 4. Among these transcription factors, NRF2 is a key negative regulator of ferroptosis [64]. The activity of NRF2 is mediated by the KEAP1/CUL3/RBX1/E3 ubiquitin ligase complex [65]. In contrast, under oxidative stress NRF2 dissociates from KEAP1 and translocates to the nucleus, where it activates the downstream expression of antioxidative response element (ARE)-containing target genes, many of which have been shown to play a role in defending against ferroptosis $[65,66]$. Interestingly, activation of NRF2 regulates iron-related genes such as FTH1, FTL1, SLC4OA1, ABCB6, and HMOX1, which in turn promotes the synthesis of GSH synthesis, limits ROS production, and regenerates NADPH $[64,66]$. The protein p53 controls ferroptosis via two distinct mechanisms. On one hand, p53 has been shown to promote ferroptosis by repressing SLC7A11 gene [52]. On the other hand, p53 has been shown to inhibit ferroptosis by negatively regulating dipeptidyl peptidase-4 (DDP-4) [67]. Depletion of ATF4 (activating transcription factor 4) has also been shown to increase erastin-induced ferroptosis and RSL3-induced ferroptosis in several cancer cell lines [68]. Finally, the Hippo pathway promotes ferroptosis by transcriptionally regulating the TFRC (which encodes TFR1) and ACSL4 genes [69]. Thus, a wide range of transcription factors appear to coordinate cellular sensitivity to ferroptosis.

\section{Ferroptosis triggers acute liver injury}

ALI is characterized by a rapid decline in hepatocyte function in patients without evidence of pre-existing liver disease [70]. In the majority of cases, ALI results from hepatotoxicity due to drugs, alcohol, ischemia/reperfusion injury (IRI), or viral hepatitis [70]. Among the long list of drugs that can cause hepatotoxicity, the canonical nonsteroidal anti-inflammatory drug acetaminophen ( $\mathrm{N}$ acetyl-para-aminophenol, or APAP) has probably been the most thoroughly documented. Lőrincz et al. [71] reported that ferroptosis occurs in APAP-induced hepatic injury, showing that ferroptosis inhibitors such as ferrostatin-1 (Fer-1) provided moderate protection against APAP-induced death in primary mouse hepatocytes. Recently, Yamada et al. [72] reported that ferroptosis occurs in a mouse model of APAP-induced acute liver failure, showing elevated lipid peroxides derived from $n-6$ PUFAs. In addition, both the ferroptosis inhibitor UAMC-3203 and the VDAC1 oligomerization inhibitor VBIT-12 have been shown to reduce ferroptosis in APAP-induced ALI animal model by protecting mitochondrial function [73]. Taken together, these studies suggest that ferroptosis may serve as a therapeutic target 
for APAP-induced ALI. Nevertheless, additional data are needed, as other forms of cell death might compensate if one form-for example, ferroptosis-is targeted.

Hepatic IRI-induced ALI can occur in a wide range of clinical contexts, including liver transplantation, systemic shock, heart failure, hemorrhage, and sepsis [74]. Many studies suggest that a complex network involving various types of cell death, including apoptosis and necroptosis, contributes to hepatic IRI. Notably, both liver IRI and kidney IRI are significantly reduced by the ferroptosis inhibitor liproxstatin-1 (Lip-1), suggesting that ferroptosis plays an important pathogenic role in IRI [4]. GPX4 has also been functionally characterized as a critical protector of both hepatic and renal function; importantly, a-tocopherol was also shown to protect against excessive lipid peroxidation in Gpx4 knockout mice [75]. Recently, a study involving human subjects found that elevated serum ferritin levels in liver donors were associated with a significant higher risk of liver damage in transplantation recipients [76], and blocking ferroptosis with compounds such as Fer-1, a-tocopherol, and DFO prevented hepatic IRI [76]. Finally, the human liver is composed of several cell types, including hepatocytes, endothelial cells, Kupffer cells and other immune cell types, bile duct cells, and epithelial progenitor cells; however, the cellular composition and contribution of each cell type during $\mathrm{ALI}$ is poorly understood and warrants further study.

\section{Ferroptosis increases chronic liver injury and fibrosis}

The majority of liver fibrosis cases were attributed to chronic hepatitis $B$ virus (HBV) and/or hepatitis C virus (HCV) infection [77]. Both HBV and HCV infections alter hepcidin levels and a variety of other iron-related parameters that are closely linked to liver fibrosis [78]. A subset of immune cells, including macrophages, neutrophils, T cells, and B cells, have been implicated in ferroptosis or are intrinsically sensitive to ferroptosis due to changes in gene expression during maturation, which can significantly affect both innate and adaptive immunity, as recently reviewed in detail by Chen et al. [79].

Whether ferroptosis plays a role in the progression of HBV/HCV infection and infection-related liver diseases such as fibrosis remains an open question. Recently, Yamane et al. [80] reported that ferroptosis plays an important role in HCV replication. Interestingly, ferroptosis inhibitors reduce the antiviral activity of direct-acting antiviral medications (DAAs) against HCV [80]. In addition, the enzyme fatty acid desaturase 2 (FADS2) converts oleate to Mead acid and other highly unsaturated fatty acids, which act as a key regulator of HCV replication by promoting lipid peroxidation [80]. Altogether, these important findings may pave the way to developing novel antiviral strategies for treating infectious liver disease.

Excess iron has long been postulated as a risk factor for developing liver fibrosis and cirrhosis, and recently direct evidence has emerged with respect to the pathogenic role of ferroptosis in iron overload-induced liver damage and fibrosis [14]. However, the precise function of iron-regulating proteins in the development of liver fibrosis remains poorly understood. From this perspective, it is interesting to note that we recently reported that transferrin plays an important role in both high dietary iron-induced liver fibrosis and carbon tetrachloride $\left(\mathrm{CCl}_{4}\right)$-induced liver fibrosis in mice, and inhibiting ferroptosis potently prevented these effects; in addition, we found that clinical data support the role of transferrin in protecting against liver fibrosis by blocking ferroptosis, providing a possible therapeutic target for preventing ferroptosis-induced liver fibrosis [26].

The transdifferentiation of hepatic stellate cells (HSCs) into matrix-producing myofibroblasts is considered a key step in the development of liver fibrosis [81]. Recent studies demonstrate the potential of inducing ferroptosis in HSCs using magnesium isoglycyrrhizinate, artesunate, or artemether as a therapeutic strategy designed to mitigate the development of liver fibrosis $[82,83]$. Moreover, certain regulators of ferroptosis in HSCs, including p53, ELAV-like protein 1 (ELAVL1), and zinc finger protein 36 (ZFP36), have been reported as promising targets in preventing liver fibrosis [84, 85]. Taken together, these studies indicate that inducing ferroptosis in HSCs may represent a viable strategy for treating and/or preventing liver fibrosis; however, a remaining challenge is the ability to selectively induce ferroptosis in HSCs with minimal effects on healthy hepatocytes.

\section{Ferroptosis and steatohepatitis}

Steatosis and steatohepatitis are two pathological manifestations of liver disease. Ferroptosis was recently associated with the onset of inflammation in steatohepatitis in the early stages of NASH in mouse models[13]. Additional studies support the notion that ferroptosis and lipid metabolic disorders play an essential role in the progression of NASH, while-conversely-inhibiting ferroptosis significantly decreases the severity of NASH $[86,87]$. Moreover, $12 / 15$-lipoxygenase has been functionally connected to hydroperoxy-phosphatidylethanolamine-related ferroptosis [88]. Recently, arachidonate 12-lipoxygenase (ALOX-12) promotes the progression NASH via its interaction with acetyl-CoA carboxylase 1 (ACC1) $[89,90]$. However, whether ferroptosis plays a role in ALOX$12-$ mediated NASH remains an open question. Taken together, these results suggest that ferroptosis may serve as a potential therapeutic target in patients with NASH. Future studies are needed to identify the precise molecular mechanisms underlying ferroptosis and lipid metabolism in the pathogenesis of NASH.

\section{Targeting ferroptosis to treat HCC}

Globally, HCC is the third leading cause of cancer-related death. Since the approval of sorafenib, the only first-line therapy for advanced HCC. Altered regulation of iron metabolism has been shown to play a role in the pathogenesis of HCC, particularly in patients who present with $\mathrm{NASH}$, hemochromatosis, or thalassemia [91, 92]. High intake of dietary iron can also increase the risk of $\mathrm{HCC}$, suggesting that high iron content may promote the development of HCC [93]. Moreover, a growing body of evidence supports the notion that activating ferroptosis may potently inhibit the growth of HCC cells, thus providing a scientific rationale for targeting ferroptosis as a novel therapeutic strategy for HCC.

Several studies using HCC cell lines suggests that ferroptosis may serve as the principal mechanism underlying the anticancer effect of sorafenib via suppressing the cystine/glutamate antiporter SLC7A11 [94-96]. Interestingly, retinoblastoma (Rb)-negative HCC cells are more susceptible to sorafenib-induced cell death compared to Rb-expressing HCC cells [97]. It should be noted that a recent study found that sorafenib was not a bona fide inducer of ferroptosis based on screening results using a panel of cancer cell lines [98]. Another recent study found that LIFR (leukemia inhibitory factor receptor) sensitized HCC cell lines to sorafenibinduced ferroptosis both in vitro and in vivo, whereas loss of LIFR expression confers resistance to sorafenib-induced ferroptosis [99]. These contradictory results might be attributed to different cellular contexts such as the expression levels of LIFR and/or other undefined genes/pathways related to the sensitivity of sorafenib.

Recently, IFNy was used to sensitize HCC cells to ferroptosis by suppressing system $x_{c}^{-}$via activation of the JAK/STAT signaling pathway, thus providing new insights into the feasibility of using IFNy to induce ferroptosis in treating HCC [100]. In addition, the progression of HCC was recently linked to the circ0097009/miR1261/SLC7A11 axis [101]. Interestingly, suppressing FTH renders HCC cells more sensitive to both RSL3- and iron-induced ferroptosis. Conversely, HCC cells with increased extracellular lactate levels are more resistant to ferroptosis induced by RSL3 and erastin [102]. Taken together, these studies suggest that inducing ferroptosis may serve as promising strategy for treating HCC. 
Table 1. Genetic and pharmacologic inducers of ferroptosis.

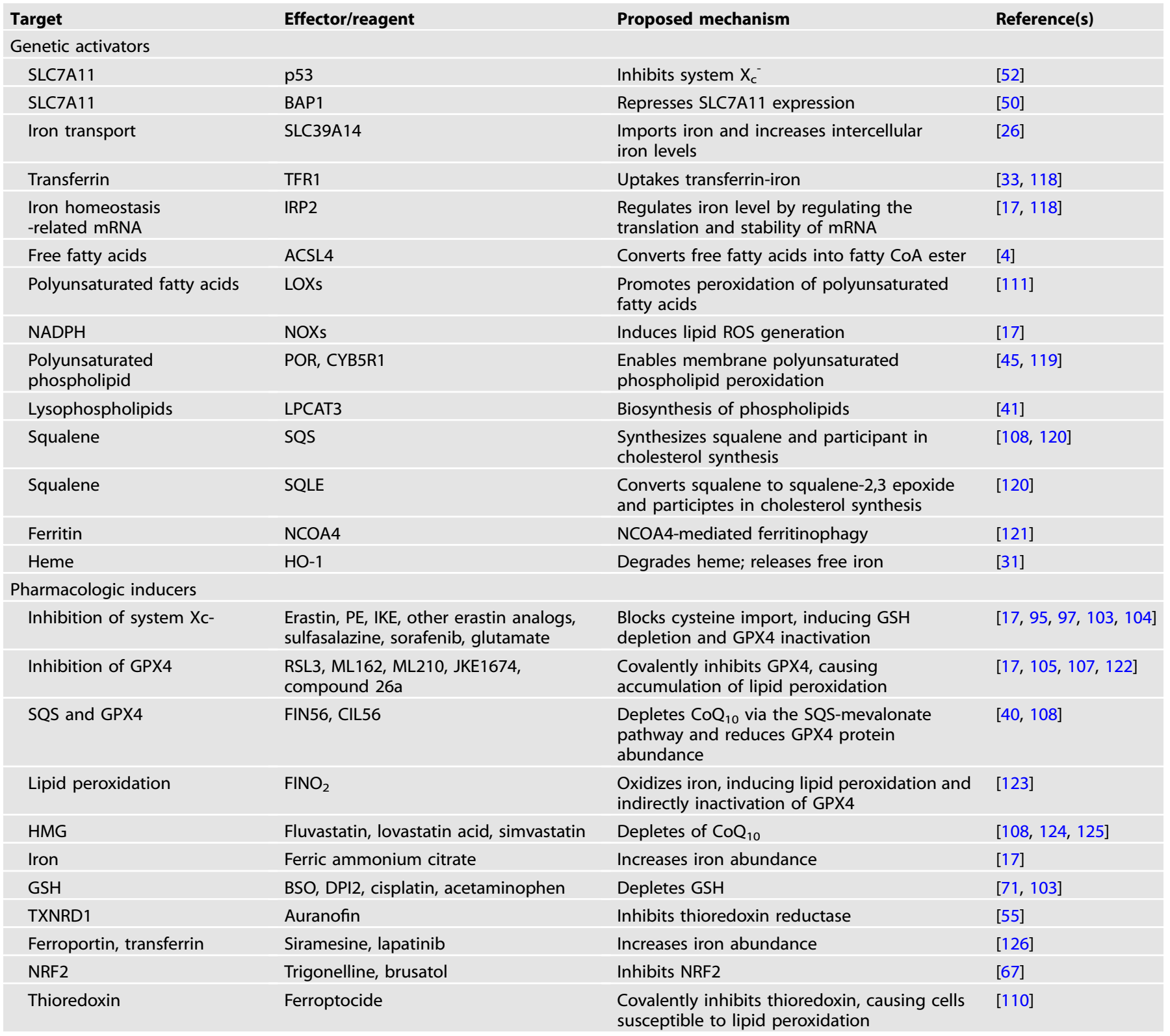

ACSL4 acyl-CoA synthetase long-chain family member 4, BAP1 BRCA1-associated protein 1, CoQ 10 ubiquinone, CYB5R1 NADPH-cytochrome P450 reductase chrome b5 reductase, GPX4 glutathione peroxidase 4, GSH glutathione, HMG 3-hydroxy-3-methylglutaryl, HO-1 heme oxygenase 1, IKE imidazole ketone erastin, IRP2 iron-regulatory protein 2, LOXs lipoxygenases, LPCAT3 lysophosphatidylcholine acyltransferase 3, NADPH nicotinamide adenine dinucleotide phosphate, NCOA4 nuclear receptor coactivator 4, NOXs NADPH oxidases, NRF2 nuclear factor erythroid 2-related factor 2, PE piperazine erastin, POR NADPH-cytochrome P450 reductase, ROS reactive oxidant species, RSL3 (1S, 3R-)RSL3, SLC7A11 solute carrier family 7 member 11, SLC39A14 solute carrier family 39 member 14 , SQLE squalene epoxidase, SQS squalene synthase, TFR1 transferrin receptor 1, TXNRD1 thioredoxin reductase 1.

\section{Therapeutic opportunities}

Ferroptosis inducers. Many ferroptosis inducers and ferroptosisrelated genes and pathways have been discovered [7] (Table 1). In addition to genetic regulators, intracellular iron accumulation, excessive lipid peroxidation, and small molecules can also trigger ferroptosis [6, 17]. Thus, ferroptosis-inducing compounds (FINs) are widely accepted as a promising approach for developing novel cancer agents. Based on their mode of action, FINs are categorized into four classes. Class I FINs activate ferroptosis by depleting intracellular GSH by inhibiting system $X_{c}^{-}$ $[17,95,103,104]$. Class II FINs induce ferroptosis by directly inactivating GPX4, such as RSL3 and ML162, which covalently bind to GPX4 to suppress its function, leading to an accumulation of toxic lipid peroxides and inducing ferroptosis [17, 105]. Notably, persistent (i.e., drug-tolerant) cancer cells are particularly vulnerable to GPX4 inhibitors. Moreover, efforts are currently being made to develop GPX4-selective covalent inhibitors with minimal toxicity and better tolerance profiles [106, 107]. Class III FINs such as FIN56 act by indirect inhibiting and inactivating GPX4 via the squalene synthase-mevalonate pathway, which participates in the mitochondrial electron transport chain and also functions as endogenous lipophilic antioxidants [40, 108]. Finally, class IV FINs induce ferroptosis by causing iron overload or by activating HO-1 [109]. 
Table 2. Genetic and pharmacologic inhibitors of ferroptosis.

\section{Target}

Genetic inhibitors

\begin{tabular}{|c|c|}
\hline SLC7A11 & NRF2 \\
\hline NRF2 & KEAP1 \\
\hline Lipid peroxides & GPX4 \\
\hline Lipid peroxides & TXNRD1 \\
\hline Cystine & SLC7A11 \\
\hline System Xc- & $\mathrm{CD} 44 \mathrm{v}$ \\
\hline Actin dynamic & HSPB1 \\
\hline Iron storage & FTH1 \\
\hline Iron metabolism & STEAP3 \\
\hline Iron transport & FPN \\
\hline Iron transport & CISD1 \\
\hline Iron metabolism genes & FANCD2 \\
\hline $\mathrm{BH}_{4}$ synthesis & $\mathrm{GCH} 1$ \\
\hline $\begin{array}{l}\text { S-adenosyl } \\
\text { homocysteine hydrolase }\end{array}$ & DJ-1 \\
\hline $\mathrm{CoQ}_{10}$ & FSP1 \\
\hline $\mathrm{CoQ}_{10}$ & $\mathrm{DHODH}$ \\
\hline $\begin{array}{l}\text { PI3K-AKT-mTOR } \\
\text { signaling pathway }\end{array}$ & mTORC1 \\
\hline SHP1-NF-кB- LCN2 & LIFR \\
\hline
\end{tabular}

Pharmacologic inhibitors Iron chelators

Lipophilic antioxidation

LOX inhibitors

Amino acid metabolism

Lipid peroxidation

Protein synthesis

GPX4

ACSL4

DPP4

Selenoproteins

NRF2

KEAP1

XNRD1

LC7A11

CD44

HSPB1

TEAP3

PN

FANCD2

$\mathrm{GCH} 1$ cyclipirox (D-PUFAs)
Effector/reagent

Deferoxamine, deferoprone, deferasirox,

Fer-1, Lip-1, UAMC-3203, TEMPO, MitoTEMPO, $\alpha$-tocopherol, trolox, BTH, $\mathrm{CoQ}_{10}$, idebenone, $\mathrm{BH}_{4}$, bazedoxifene, 2-(1-(4-(4-methylpiperazin1-yl)phenyl)ethyl)-10H-phenothiazine

Zileuton, AA-861, PD-146176

Glutathione, $\mathrm{N}$-acetylcysteine, $\beta$-mercaptoethanol

Deuterated polyunsaturated fatty acids

Cycloheximide

\section{PKUMDL-LC-101-D04}

Rosiglitazone, pioglitazone, troglitazone

Vildagliptin, linagaliptin, alogliptin

Selenium, methylselenocysteine, selenocystamine
Proposed mechanism

Reference(s)

Activates antioxidant genes; Upregulates SLC7A11

Stabilizes and regulates NRF2

Eliminates phospholipid hydroperoxides

Eliminates phospholipid hydroperoxides

Component of system $\mathrm{X}_{\mathrm{c}}^{-}$; Antiporter for cystine

Binds to SLC7A11 to stabilize system $\mathrm{X}_{\mathrm{c}}^{-}$

Regulates iron uptake and GPX4 abundance

Subunit of intercellular iron-storage protein

Metalloreductase converting $\mathrm{Fe}^{3+}$ to $\mathrm{Fe}^{2+}$

Exports iron and reduces intercellular iron accumulation

Inhibits mitochondrial iron uptake and respiratory capacity

Regulates iron metabolism and lipid peroxidation

Involved in $\mathrm{BH}_{4}$ synthesis

Determines the formation of the S-adenosyl homocysteine hydrolase tetramer and its enzymatic activity

Converts $\mathrm{CoQ}_{10}$ to $\mathrm{CoQ}_{10} \mathrm{H}_{2}$, reducing membrane phospholipid peroxidation

Converts $\mathrm{CoQ}_{10}$ to $\mathrm{CoQ}_{10} \mathrm{H}_{2}$ in mitochondrial

Regulates amino acid metabolism and upregulates SREBP1/SCD1-mediated lipogenesis

Suppresses NF- $\mathrm{kB}$ signaling through SHP1, leading to downregulation of LCN2

Chelate iron and block irondependent lipid peroxidation

Inhibit lipid peroxidation

$17,23,61,115,136]$

Suppress LOXs and blocks LOXinduced lipid peroxidation

Recover intracellular cysteine

Suppress the initiation and propagation of lipid peroxidation

Blocks ferroptosis induced by system $\mathrm{X}_{\mathrm{c}}^{-}$

Activates GPX4 by allosteric activity

Inhibits ACSL4

Suppress DPP4-dependent lipid peroxidation

Replenishes of GPX4 and other selenoproteins
$[4,15-$

$[111,113,137]$

[17]

$[14,17]$

$[127,130,131]$

[132]

$28,133]$

$[56,139,140]$ 
Table 2. continued

Target
N/A

Effector/reagent
Necrostatin-1, necrostatin-1f

\section{Proposed mechanism \\ Suppress ferroptosis in a RIPK1- \\ independent manner}

Reference(s)

$[4,114]$

ACSL4 acyl-CoA synthetase long-chain family member 4, $A K T$ AKT serine/threonine kinase, $B T H$ butylated hydroxytoluene, $B H_{4}$ tetrahydrobiopterin, $C D 44 v$ CD44 variant, CISD1 CDGSH iron-sulfur domain-containing protein $1, C_{0} Q_{10}$ ubiquinone, $C_{0} Q_{10} H_{2}$ ubiquinol, $D H O D H$ dihydroorotate dehydrogenase, $D P P 4$ dipeptidyl peptidase-4, FANCD2 Fanconi anemia complementation group d2, Fer-1 ferrostatin-1, FPN ferroportin, FTH1 ferritin heavy chain 1, FSP1 ferroptosis suppressor protein 1, GCH1 guanosine triphosphate cyclohydrolase 1, GPX4 glutathione peroxidase 4, HSPB1 heat shock protein b1, KEAP1 kelch like ECH associated protein 1, LCN2 lipocalin 2, LIFR leukemia inhibitory factor receptor, Lip-1 liproxastatin-1, LOX lipoxygenases, mTORC1 mechanistic target of rapamycin complex 1, NRF2 nuclear factor erythroid 2-related factor 2, PI3K phosphoinositide-3-kinase, RIPK1 receptor interacting protein kinase 1, SCD1 stearoyl-CoA desaturase, SHP1 SH2-containing protein tyrosine phosphatase, SLC7A11 solute carrier family 7 member 11 , SREBP1 sterol regulatory element-binding protein 1, STEAP3 sixtransmembrane epithelial antigen of prostate 3, TXNRD1 thioredoxin reductase 1, N/A not applicable.

Many ferroptosis inducers do not fall into any of these four FIN classes and are summarized in Table 2. For example, the compound ferroptocide was recently identified as a potent ferroptosis agonist that covalently binds to thioredoxin (TXN), a 12-kDa ubiquitous oxidoreductase in the thioredoxin antioxidant system [110]. This binding is similar to the mechanism used by other ferroptosis inducers such as RSL3 and its analogs $[103,106,107,111]$. The chloroacetamide-mediated activity of RSL3 and its analogs results in low target selectivity, as RSL3 can covalently modify a subset of non-GPX4 selenoproteins at the active site [106, 107, 112]. Similarly, the putative off-target effects of this compound may be attributed to its extensive covalent interactions with the proteome in ferroptocide-treated cells, which could be optimized by improving the selectivity for ferroptosis and proteins of interest.

Ferroptosis inhibitors. Ferroptosis can be inhibited using three main approaches (Table 2): iron chelation, preventing lipid peroxidation, and scavenging lipid peroxides. Of these three strategies, iron chelators and lipophilic antioxidants are widely accepted ferroptosis inhibitors. Iron chelators such as deferoxamine (DFO), deferiprone, and ciclopirox chelate iron and prevent the propagation of lipid peroxidation by limiting the Fenton reaction [17]. To date, a small handful of iron chelators have been approved by the FDA or have undergone clinical trials to treat iron overload-related diseases, transplantation, organ injury, and HCC. Lipophilic antioxidants, including a-tocopherol, Fer-1, and Lip-1, function as radical scavengers to reduce lipid peroxides and are effective at blocking ferroptosis [4, 17]. In mouse models of diseases such as ALI, liver fibrosis, NAFLD, and NASH, treatment with lipophilic antioxidants was shown to prevent disease progression and extend life expectancy. However, several limitations may preclude the use of Fer- 1 and Lip-1 in clinical settings, including their possible unsatisfactory pharmacokinetics and pharmacodynamics profiles. For example, the biological half-life of Fer-1 is only a few minutes, which is generally unacceptable for clinical applications. With respect to the long-term use of ferroptosis inhibitors in patients with chronic conditions, previously undefined side effects may also appear in these patients. Notably, ACSL4 inhibitors such as rosiglitazone, troglitazone, and pioglitazone were previously reported to inhibit ferroptosis in a lipoxygenase-dependent manner; however, this finding was challenged by the finding that these ACSL4 inhibitors have inherent antioxidant activity [113].

Importantly, necrostatin-1 (Nec-1), a widely used inhibitor of necroptosis, has been suggested to inhibit both necroptosis and ferroptosis. For example, Nec-1f, a highly selective inhibitor of RIPK1 (receptor interacting protein kinase 1) has been shown to simultaneously inhibit necroptosis and ferroptosis in primary kidney tubules and mouse cardiac transplantation models [114]. Future studies are warranted to identify the mechanism underlying this dual inhibition as well as potential targets for treating pathological conditions associated with both necroptosis and ferroptosis. It is also interesting to note that Nec-1 has a better pharmacokinetics profile than Fer- 1 , and the maximum concentration of Nec-1f in tissues is relatively well tolerated [114-116], suggesting that Nec-1f may have better translational potential than Fer-1.

An number of ferroptosis-inhibiting compounds have been identified, including-but not limited to-beta-mercaptoethanol, selenium, cycloheximide, dopamine, and glutaminolysis inhibitors (Table 2). To the best of our knowledge, however, no clinical trials have been performed to test ferroptosis inhibitors in liver disease [3]. Given our limited understanding of ferroptosis-related pathways and regulatory mechanisms in a wide range of disease conditions, many questions remain. Regardless, ferroptosis inhibitors have been shown to be efficacious, at least in preclinical studies. Thus, future studies should focus on developing more potent ferroptosis inhibitors with improved drug properties, ideally leading to clinical testing.

\section{Future directions and perspectives}

Given the emerging role of ferroptosis in the pathogenesis of various liver diseases, ferroptosis serves as a promising therapeutic target (Fig. 5). Further studies are warranted in order to determine the precise role of ferroptosis at the cellular, tissue, and systemic levels. In the context of liver disease, more evidence is needed in order to provide a complete view of the dynamic processes by which ferroptosis drives the initiation of inflammation leading to liver fibrosis, cirrhosis, and-ultimately-carcinogenesis.

Based on well-established ferroptosis targets, significant effort has gone into drug design, such as designing allosteric activators of GPX4 [117]. Screening existing compound libraries and FDAapproved drugs will also likely help identify novel ferroptosis modulators and their underlying mechanisms. In this respect, high-throughput functional screening, automation, and artificial intelligence-based approaches may accelerate the development of ferroptosis-targeted drugs. Moreover, understanding the structure-function relationship and optimizing small molecules may help accelerate the development of new drugs. Indeed, an optimized Fer-1 molecule with an improved ADME (absorption, distribution, metabolism, and excretion) profile is expected to move into preclinic and clinic trials [115]. In addition, pharmaceutical studies are needed in order to successfully deliver the drugs to specific organs such as the liver with maximum safety and efficacy in a wide range of disease settings.

Although much progress has been made in our understanding of the pathological roles of ferroptosis in liver diseases, several critical questions remain to be addressed for further clinical development of ferroptosis-targeted therapies. First, what physiological role, if any, does ferroptosis play in the liver? Second, can we identify reliable, sensitive biomarkers of ferroptosis in liver disease? Third, when should we target ferroptosis in specific pathological liver conditions and/or disease stages? Fourth, in treating liver cancer, can we activate ferroptosis specifically in cancer cells without affecting healthy cells? Tackling the key 


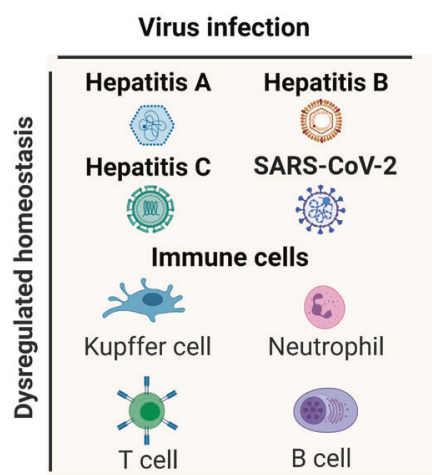

Anti-ferroptosis

Clinical medications

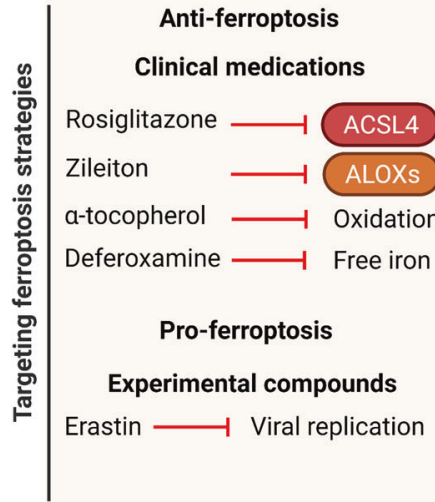

Promote ferroptosis

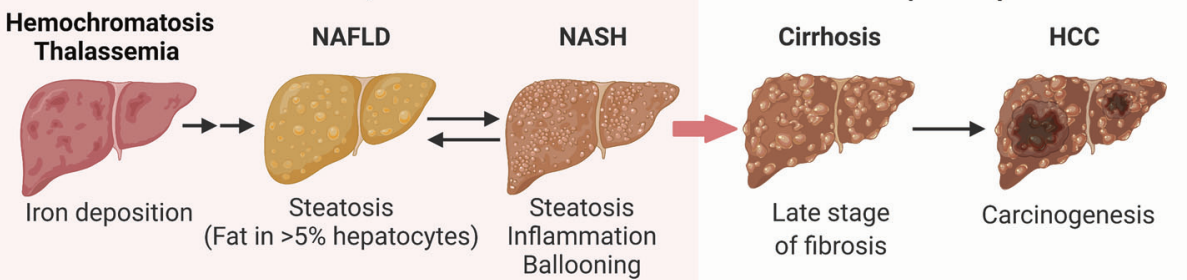

\section{Escape ferroptosis}

of fibrosis

\section{Anti-ferroptosis \\ Clinical medications}

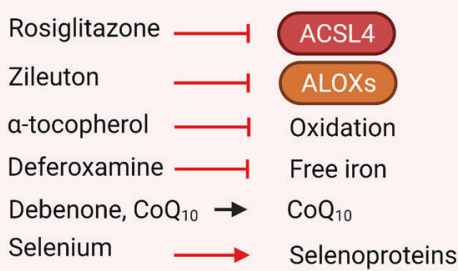

Experimental compounds

Fer-1, Lip-1 MitoTEMPO

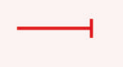

Free radical

Fig. 5 Strategies for targeting ferroptosis in treating liver disease. Dysregulated iron metabolism has been implicated in the development of a variety of hepatic diseases, including viral infectious liver disease, iron-overload disease, and the progression from NAFLD to HCC. These liver diseases can be treated using either ferroptosis inhibitors (middle panel) or ferroptosis inducers (right panel). Infectious liver diseases can be treated using ferroptosis inhibitors and ferroptosis inducers for host cells and viruses respectively (left panel). GPX4, glutathione peroxidase 4; ACSL4, acyl-CoA synthetase long-chain family member 4; ALOXs, arachidonate lipoxygenases; CoQ 10 , ubiquinone; Fer-1, ferrostatin-1; FSP1, ferroptosis suppressor protein 1; HCC, hepatocellular carcinoma; Lip-1, liproxstatin-1; NAFLD, non-alcoholic fatty liver disease; NASH, nonalcoholic steatohepatitis; SLC7A11, solute carrier family 7 member 11; TXNRD1, thioredoxin reductase 1.

scientific issues discussed in this review will improve our understanding of the precise role that ferroptosis plays in various pathophysiological liver conditions, thus providing a new scientific rationale for targeting ferroptosis in order to prevent and treat liver disease.

\section{REFERENCES}

1. Asrani SK, Devarbhavi H, Eaton J, Kamath PS. Burden of liver diseases in the world. J Hepatol. 2019;70:151-71.

2. Luedde T, Kaplowitz N, Schwabe RF. Cell death and cell death responses in liver disease: mechanisms and clinical relevance. Gastroenterology. 2014;147:765-83.

3. Schwabe RF, Luedde T. Apoptosis and necroptosis in the liver: a matter of life and death. Nat Rev Gastroentero Hepat. 2018;15:738-52.

4. Friedmann Angeli JP, Schneider M, Proneth B, Tyurina YY, Tyurin VA, Hammond VJ, et al. Inactivation of the ferroptosis regulator $\mathrm{Gpx} 4$ triggers acute renal failure in mice. Nat Cell Biol. 2014;16:1180-91.

5. Gautheron J, Gores GJ, Rodrigues CMP. Lytic cell death in metabolic liver disease. J Hepatol. 2020;73:394-408.

6. Stockwell BR, Friedmann Angeli JP, Bayir H, Bush Al, Conrad M, Dixon SJ, et al. Ferroptosis: a regulated cell death nexus linking metabolism, redox biology, and disease. Cell. 2017;171:273-85.

7. Xie $Y$, Hou W, Song $X, Y u$ Y, Huang J, Sun $X$, et al. Ferroptosis: process and function. Cell Death Differ. 2016;23:369-79.

8. Zheng J, Conrad M. The metabolic underpinnings of ferroptosis. Cell Metab. 2020;32:920-37.

9. Nie J, Lin B, Zhou M, Wu L, Zheng T. Role of ferroptosis in hepatocellular carcinoma. J Cancer Res Clin Oncol. 2018;144:2329-37.

10. Shojaie L, lorga A, Dara L. Cell death in liver diseases: a review. Int J Mol Sci. 2020;21:9682.

11. Cusi K. Role of obesity and lipotoxicity in the development of nonalcoholic steatohepatitis: pathophysiology and clinical implications. Gastroenterology. 2012;142:711-U109.
12. Masoodi M, Gastaldelli A, Hyötyläinen T, Arretxe $E$, Alonso C, Gaggini $M$, et al. Metabolomics and lipidomics in NAFLD: biomarkers and non-invasive diagnostic tests. Nat Rev Gastroenterol Hepatol. 2021;18:835-56.

13. Tsurusaki S, Tsuchiya $Y$, Koumura T, Nakasone M, Sakamoto T, Matsuoka M, et al. Hepatic ferroptosis plays an important role as the trigger for initiating inflammation in nonalcoholic steatohepatitis. Cell Death Dis. 2019;10:449.

14. Wang $\mathrm{H}, \mathrm{An} \mathrm{P}, \mathrm{Xie} \mathrm{E}, \mathrm{Wu} \mathrm{Q}$, Fang $\mathrm{X}$, Gao H, et al. Characterization of ferroptosis in murine models of hemochromatosis. Hepatology. 2017;66:449-65.

15. Doll S, Freitas FP, Shah R, Aldrovandi $M$, da Silva MC, Ingold $I$, et al. FSP1 is a glutathione-independent ferroptosis suppressor. Nature. 2019;575:693-8.

16. Bersuker K, Hendricks JM, Li Z, Magtanong L, Ford B, Tang PH, et al. The CoQ oxidoreductase FSP1 acts parallel to GPX4 to inhibit ferroptosis. Nature. 2019;575:688-92.

17. Dixon SJ, Lemberg KM, Lamprecht MR, Skouta R, Zaitsev EM, Gleason CE, et al. Ferroptosis: an iron-dependent form of nonapoptotic cell death. Cell. 2012;149:1060-72.

18. Shimada K, Hayano M, Pagano NC, Stockwell BR. Cell-line selectivity improves the predictive power of pharmacogenomic analyses and helps identify NADPH as biomarker for ferroptosis sensitivity. Cell Chem Biol. 2016;23:225-35.

19. Yang WS, Stockwell BR. Ferroptosis: death by lipid peroxidation. Trends Cell Biol. 2016;26:165-76.

20. Younossi ZM, Koenig AB, Abdelatif D, Fazel Y, Henry L, Wymer M. Global epidemiology of nonalcoholic fatty liver disease-Meta-analytic assessment of prevalence, incidence, and outcomes. Hepatology. 2016;64:73-84.

21. Rinella $M$, Charlton $M$. The globalization of nonalcoholic fatty liver disease: Prevalence and impact on world health. Hepatology. 2016;64:19-22.

22. Sun $Y$, Zheng $Y$, Wang $C$, Liu Y. Glutathione depletion induces ferroptosis, autophagy, and premature cell senescence in retinal pigment epithelial cells. Cell Death Dis. 2018;9:753.

23. Conlon M, Poltorack CD, Forcina GC, Armenta DA, Mallais M, Perez MA, et al. A compendium of kinetic modulatory profiles identifies ferroptosis regulators. Nat Chem Biol. 2021;17:665-74.

24. Ganz T. Hepcidin, a key regulator of iron metabolism and mediator of anemia of inflammation. Blood. 2003;102:783-8. 
25. Fillebeen C, Charlebois E, Wagner J, Katsarou A, Mui J, Vali H, et al. Transferrin receptor 1 controls systemic iron homeostasis by fine-tuning hepcidin expression to hepatocellular iron load. Blood. 2019;133:344-55.

26. Yu Y, Jiang $L$, Wang $H$, Shen $Z$, Cheng $Q$, Zhang $P$, et al. Hepatic transferrin plays a role in systemic iron homeostasis and liver ferroptosis. Blood. 2020;136:726-39.

27. Jenkitkasemwong S, Wang C-Y, Coffey R, Zhang W, Chan A, Biel T, et al. SLC39A14 is required for the development of hepatocellular iron overload in murine models of hereditary hemochromatosis. Cell Metab. 2015;22:138-50.

28. Zhang Z, Zhang F, Guo X, An P, Tao Y, Wang F. Ferroportin1 in hepatocytes and macrophages is required for the efficient mobilization of body iron stores in mice. Hepatology. 2012;56:961-71.

29. Wang F, Paradkar PN, Custodio AO, McVey Ward D, Fleming MD, Campagna D, et al. Genetic variation in Mon1a affects protein trafficking and modifies macrophage iron loading in mice. Nat Genet. 2007;39:1025-32.

30. Jiang L, Wang J, Wang $K$, Wang $H$, Wu Q, Yang $C$, et al. RNF217 regulates iron homeostasis through its E3 ubiquitin ligase activity by modulating ferroportin degradation. Blood. 2021;138:689-705.

31. Fang $X$, Wang $H$, Han $D$, Xie $E$, Yang $X$, Wei J, et al. Ferroptosis as a target for protection against cardiomyopathy. Proc Natl Acad Sci USA. 2019;116:2672-80.

32. Dixon SJ, Stockwell BR. The role of iron and reactive oxygen species in cell death. Nat Chem Biol. 2014;10:9-17.

33. Gao M, Monian P, Quadri N, Ramasamy R, Jiang X. Glutaminolysis and transferrin regulate ferroptosis. Mol Cell. 2015;59:298-308.

34. Yuan H, Li X, Zhang X, Kang R, Tang D. CISD1 inhibits ferroptosis by protection against mitochondrial lipid peroxidation. Biochem Biophys Res Commun. 2016;478:838-44.

35. Hou W, Xie Y, Song X, Sun X, Lotze MT, Zeh HJ 3rd, et al. Autophagy promotes ferroptosis by degradation of ferritin. Autophagy. 2016;12:1425-8.

36. Protchenko O, Baratz E, Jadhav S, Li F, Shakoury-Elizeh M, Gavrilova O, et al. Iron chaperone poly $\mathrm{rC}$ binding protein 1 protects mouse liver from lipid peroxidation and steatosis. Hepatology. 2021;73:1176-93.

37. Kagan VE, Mao G, Qu F, Angeli JP, Doll S, Croix CS, et al. Oxidized arachidonic and adrenic PEs navigate cells to ferroptosis. Nat Chem Biol. 2017;13:81-90.

38. Vriens K, Christen S, Parik S, Broekaert D, Yoshinaga K, Talebi A, et al. Evidence for an alternative fatty acid desaturation pathway increasing cancer plasticity. Nature. 2019;566:403-6.

39. Lee H, Zandkarimi F, Zhang Y, Meena JK, Kim J, Zhuang L, et al. Energy-stressmediated AMPK activation inhibits ferroptosis. Nat Cell Biol. 2020;22:225-34.

40. Dixon SJ, Winter GE, Musavi LS, Lee ED, Snijder B, Rebsamen M, et al. Human haploid cell genetics reveals roles for lipid metabolism genes in nonapoptotic cell death. ACS Chem Biol. 2015:10:1604-9.

41. Doll S, Proneth B, Tyurina YY, Panzilius E, Kobayashi S, Ingold I, et al. ACSL4 dictates ferroptosis sensitivity by shaping cellular lipid composition. Nat Chem Biol. 2017;13:91-8.

42. Gaschler MM, Stockwell BR. Lipid peroxidation in cell death. Biochem Biophys Res Commun. 2017;482:419-25.

43. Zorov DB, Juhaszova M, Sollott SJ. Mitochondrial reactive oxygen species (ROS) and ROS-induced ROS release. Physiol Rev. 2014;94:909-50.

44. Laneuville O, Breuer DK, Xu N, Huang ZH, Gage DA, Watson JT, et al. Fatty acid substrate specificities of human prostaglandin-endoperoxide $\mathrm{H}$ synthase- 1 and -2. Formation of 12-hydroxy-(9Z, 13E/Z, 15Z)- octadecatrienoic acids from alphalinolenic acid. J Biol Chem. 1995;270:19330-6.

45. Zou Y, Li H, Graham ET, Deik AA, Eaton JK, Wang W, et al. Cytochrome P450 oxidoreductase contributes to phospholipid peroxidation in ferroptosis. Nat Chem Biol. 2020;16:302-9.

46. Feng $\mathrm{H}$, Stockwell BR. Unsolved mysteries: How does lipid peroxidation cause ferroptosis? PLoS Biol. 2018;16:e2006203.

47. Gao M, Yi J, Zhu J, Minikes AM, Monian P, Thompson CB, et al. Role of Mitochondria in Ferroptosis. Molecular Cell. 2019;73:354-63.

48. Torii S, Shintoku R, Kubota C, Yaegashi M, Torii R, Sasaki M, et al. An essential role for functional lysosomes in ferroptosis of cancer cells. Biochem $\mathrm{J}$. 2016;473:769-77.

49. Belalcázar AD, Ball JG, Frost LM, Valentovic MA, Wilkinson J. Transsulfuration is a significant source of sulfur for glutathione production in human mammary epithelial cells. ISRN Biochem. 2014;2013:637897.

50. Zhang Y, Shi J, Liu X, Feng L, Gong Z, Koppula P, et al. BAP1 links metabolic regulation of ferroptosis to tumour suppression. Nat Cell Biol. 2018;20:1181-92.

51. Dai E, Meng L, Kang R, Wang X, Tang D. ESCRT-III-dependent membrane repair blocks ferroptosis. Biochem Biophys Res Commun. 2020;522:415-21.

52. Jiang L, Kon N, Li T, Wang S-J, Su T, Hibshoosh H, et al. Ferroptosis as a p53mediated activity during tumour suppression. Nature. 2015;520:57-62.

53. Liu T, Jiang L, Tavana O, Gu W. The deubiquitylase OTUB1 mediates ferroptosis via stabilization of SLC7A11. Cancer Res. 2019;79:1913-24.
54. Mandal PK, Seiler A, Perisic T, Kölle P, Banjac Canak A, Förster H, et al. System x (c)- and thioredoxin reductase 1 cooperatively rescue glutathione deficiency. J Biol Chem. 2010;285:22244-53.

55. Yang $L$, Wang $H$, Yang $X, W u ~ Q, ~ A n ~ P$, Jin $X$, et al. Auranofin mitigates systemic iron overload and induces ferroptosis via distinct mechanisms. Signal Transduct Target Ther. 2020;5:138.

56. Ingold I, Berndt C, Schmitt S, Doll S, Poschmann G, Buday K, et al. Selenium utilization by GPX4 is required to prevent hydroperoxide-induced ferroptosis. Cell. 2018;172:409-22.

57. Maiorino M, Conrad M, Ursini F. GPx4, lipid peroxidation, and cell death: discoveries, rediscoveries, and open issues. Antioxid Redox Signal. 2018;29:61-74.

58. Conrad M, Moreno SG, Sinowatz F, Ursini F, Kölle S, Roveri A, et al. The nuclear form of phospholipid hydroperoxide glutathione peroxidase is a protein thiol peroxidase contributing to sperm chromatin stability. Mol Cell Biol. 2005;25:7637-44.

59. Schneider $M$, Forster $H$, Boersma $A$, Seiler $A$, Wehnes $H$, Sinowatz $F$, et al. Mitochondrial glutathione peroxidase 4 disruption causes male infertility. FASEB J. 2009;23:3233-42.

60. Kraft VAN, Bezjian CT, Pfeiffer S, Ringelstetter L, Müller C, Zandkarimi F, et al. GTP cyclohydrolase 1/tetrahydrobiopterin counteract ferroptosis through lipid remodeling. ACS Cent Sci. 2020;6:41-53.

61. Soula M, Weber RA, Zilka O, Alwaseem H, La K, Yen F, et al. Metabolic determinants of cancer cell sensitivity to canonical ferroptosis inducers. Nat Chem Biol. 2020;16:1351-60

62. Gan B. Mitochondrial regulation of ferroptosis. J Cell Biol. 2021;220:e202105043.

63. Mao C, Liu X, Zhang Y, Lei G, Yan Y, Lee H, et al. DHODH-mediated ferroptosis defence is a targetable vulnerability in cancer. Nature. 2021;593:586-90.

64. Dodson M, Castro-Portuguez R, Zhang DD. NRF2 plays a critical role in mitigating lipid peroxidation and ferroptosis. Redox Biol. 2019;23:101107.

65. Suzuki T, Motohashi H, Yamamoto M. Toward clinical application of the Keap1Nrf2 pathway. Trends Pharm Sci. 2013;34:340-6.

66. Kerins MJ, Ooi A. The roles of NRF2 in modulating cellular iron homeostasis. Antioxid Redox Signal. 2018;29:1756-73.

67. Xie Y, Zhu S, Song X, Sun X, Fan Y, Liu J, et al. The tumor suppressor p53 limits ferroptosis by blocking DPP4 activity. Cell Rep. 2017;20:1692-704.

68. Chen D, Fan Z, Rauh M, Buchfelder M, Eyupoglu IY, Savaskan N. ATF4 promotes angiogenesis and neuronal cell death and confers ferroptosis in a xCTdependent manner. Oncogene. 2017;36:5593-608.

69. Wu J, Minikes AM, Gao M, Bian H, Li Y, Stockwell BR, et al. Intercellular interaction dictates cancer cell ferroptosis via NF2-YAP signalling. Nature. 2019;572:402-6.

70. Stravitz RT, Lee WM. Acute liver failure. Lancet. 2019;394:869-81.

71. Lorincz T, Jemnitz K, Kardon T, Mandl J, Szarka A. Ferroptosis is involved in acetaminophen induced cell death. Pathol Oncol Res. 2015;21:1115-21.

72. Yamada N, Karasawa T, Kimura H, Watanabe S, Komada T, Kamata R, et al. Ferroptosis driven by radical oxidation of $n-6$ polyunsaturated fatty acids mediates acetaminophen-induced acute liver failure. Cell Death Dis. 2020;11:144.

73. Niu B, Lei $X, X u$ Q, Ju Y, Xu D, Mao L, et al. Protecting mitochondria via inhibiting VDAC1 oligomerization alleviates ferroptosis in acetaminophen-induced acute liver injury. Cell Biol Toxicol. 2021. https://doi.org/10.1007/s10565-021-09624-x.

74. Weigand $\mathrm{K}$, Brost $\mathrm{S}$, Steinebrunner $\mathrm{N}$, Buchler $\mathrm{M}$, Schemmer $\mathrm{P}$, Muller $\mathrm{M}$. Ischemia/reperfusion injury in liver surgery and transplantation: pathophysiology. HPB Surg. 2012;2012:176723.

75. Carlson BA, Tobe R, Yefremova E, Tsuji PA, Hoffmann VJ, Schweizer U, et al. Glutathione peroxidase 4 and vitamin E cooperatively prevent hepatocellular degeneration. Redox Biol. 2016;9:22-31.

76. Yamada N, Karasawa T, Wakiya T, Sadatomo A, Ito H, Kamata R, et al. Iron overload as a risk factor for hepatic ischemia-reperfusion injury in liver transplantation: potential role of ferroptosis. Am J Transpl. 2020;20:1606-18.

77. El-Serag HB. Epidemiology of viral hepatitis and hepatocellular carcinoma. Gastroenterology. 2012;142:1264-73.

78. Kanda T, Goto T, Hirotsu Y, Moriyama M, Omata M. Molecular mechanisms driving progression of liver cirrhosis towards hepatocellular carcinoma in chronic hepatitis B and C infections: a review. Int J Mol Sci. 2019;20:1358.

79. Chen X, Kang R, Kroemer G, Tang D. Ferroptosis in infection, inflammation, and immunity. J Exp Med. 2021;218:e20210518.

80. Yamane $D$, Hayashi $Y$, Matsumoto $M$, Nakanishi $H$, Imagawa $H$, Kohara $M$, et al. FADS2-dependent fatty acid desaturation dictates cellular sensitivity to ferroptosis and permissiveness for hepatitis $C$ virus replication. Cell Chem Biol. 2021;21:00362-7.

81. Tsuchida T, Friedman SL. Mechanisms of hepatic stellate cell activation. Nat Rev Gastroenterol Hepatol. 2017;14:397-411. 
82. Sui M, Jiang $X$, Chen J, Yang H, Zhu Y. Magnesium isoglycyrrhizinate ameliorates liver fibrosis and hepatic stellate cell activation by regulating ferroptosis signaling pathway. Biomed Pharmacother. 2018;106:125-33.

83. Kong Z, Liu R, Cheng Y. Artesunate alleviates liver fibrosis by regulating ferroptosis signaling pathway. Biomed Pharmacother. 2019;109:2043-53.

84. Zhang Z, Yao Z, Wang L, Ding $\mathrm{H}$, Shao J, Chen A, et al. Activation of ferritinophagy is required for the RNA-binding protein ELAVL1/HuR to regulate ferroptosis in hepatic stellate cells. Autophagy. 2018;14:2083-103.

85. Zhang Z, Guo M, Li Y, Shen M, Kong D, Shao J, et al. RNA-binding protein ZFP36/ TTP protects against ferroptosis by regulating autophagy signaling pathway in hepatic stellate cells. Autophagy. 2020;16:1482-505.

86. Li X, Wang TX, Huang X, Li Y, Sun T, Zang S, et al. Targeting ferroptosis alleviates methionine-choline deficient (MCD)-diet induced NASH by suppressing liver lipotoxicity. Liver Int. 2020;40:1378-94.

87. Qi J, Kim JW, Zhou Z, Lim CW, Kim B. Ferroptosis affects the progression of nonalcoholic steatohepatitis via the modulation of lipid peroxidation-mediated cell death in mice. Am J Pathol. 2020;190:68-81.

88. Wenzel SE, Tyurina YY, Zhao J, St Croix CM, Dar HH, Mao G, et al. PEBP1 wardens ferroptosis by enabling lipoxygenase generation of lipid death signals. Cell. 2017;171:628-41.

89. Zhang XJ, She ZG, Wang J, Sun D, Shen LJ, Xiang H, et al. Multiple omics study identifies an interspecies conserved driver for nonalcoholic steatohepatitis. Sci Transl Med. 2021;13:eabg8117.

90. Zhang XJ, Ji YX, Cheng X, Cheng $\mathrm{Y}$, Yang H, Wang J, et al. A small molecule targeting ALOX12-ACC1 ameliorates nonalcoholic steatohepatitis in mice and macaques. Sci Transl Med. 2021;13:eabg8116.

91. Datz C, Felder TK, Niederseer D, Aigner E. Iron homeostasis in the metabolic syndrome. Eur J Clin Invest. 2013;43:215-24.

92. Borgna-Pignatti C, Vergine G, Lombardo T, Cappellini MD, Cianciulli P, Maggio A, et al. Hepatocellular carcinoma in the thalassaemia syndromes. $\mathrm{Br} \mathrm{J}$ Haematol. 2004;124:114-7.

93. Fonseca-Nunes A, Jakszyn P, Agudo A. Iron and cancer risk-a systematic review and meta-analysis of the epidemiological evidence. Cancer Epidemiol Biomark Prev. 2014;23:12-31.

94. Louandre C, Ezzoukhry Z, Godin C, Barbare JC, Maziere JC, Chauffert B, et al Iron-dependent cell death of hepatocellular carcinoma cells exposed to sorafenib. Int J Cancer. 2013;133:1732-42.

95. Dixon SJ, Patel DN, Welsch M, Skouta R, Lee ED, Hayano M, et al. Pharmacological inhibition of cystine-glutamate exchange induces endoplasmic reticulum stress and ferroptosis. Elife. 2014;3:1-25.

96. Lachaier E, Louandre C, Godin C, Saidak Z, Baert M, Diouf M, et al. Sorafenib induces ferroptosis in human cancer cell lines originating from different solid tumors. Anticancer Res. 2014;34:6417-22.

97. Louandre C, Marcq I, Bouhlal H, Lachaier E, Godin C, Saidak Z, et al. The retinoblastoma $(\mathrm{Rb})$ protein regulates ferroptosis induced by sorafenib in human hepatocellular carcinoma cells. Cancer Lett. 2015;356:971-7.

98. Zheng J, Sato M, Mishima E, Sato H, Proneth B, Conrad M. Sorafenib fails to trigger ferroptosis across a wide range of cancer cell lines. Cell Death Dis. 2021;12:698

99. Yao F, Deng Y, Zhao Y, Mei Y, Zhang Y, Liu X, et al. A targetable LIFR-NF-KB-LCN2 axis controls liver tumorigenesis and vulnerability to ferroptosis. Nat Commun. 2021;12:7333.

100. Kong R, Wang N, Han W, Bao W, Lu J. IFNgamma-mediated repression of system $\mathrm{xc}(-)$ drives vulnerability to induced ferroptosis in hepatocellular carcinoma cells. J Leukoc Biol. 2021;110:301-14.

101. Lyu N, Zeng Y, Kong Y, Chen Q, Deng H, Ou S, et al. Ferroptosis is involved in the progression of hepatocellular carcinoma through the circ0097009/miR-1261/ SLC7A11 axis. Ann Transl Med. 2021;9:675.

102. Zhao $Y$, Li M, Yao X, Fei $Y$, Lin $Z$, Li Z, et al. HCAR1/MCT1 regulates tumor ferroptosis through the lactate-mediated AMPK-SCD1 activity and its therapeutic implications. Cell Rep. 2020;33:108487.

103. Yang Wan S, SriRamaratnam R, Welsch Matthew E, Shimada K, Skouta R, Viswanathan Vasanthi $S$, et al. Regulation of ferroptotic cancer cell death by GPX4 Cell. 2014;156:317-31.

104. Larraufie $M H$, Yang WS, Jiang E, Thomas AG, Slusher BS, Stockwell BR. Incorporation of metabolically stable ketones into a small molecule probe to increase potency and water solubility. Bioorg Med Chem Lett. 2015;25:4787-92.

105. Weiwer M, Bittker JA, Lewis TA, Shimada K, Yang WS, MacPherson L, et al. Development of small-molecule probes that selectively kill cells induced to express mutant RAS. Bioorg Med Chem Lett. 2012;22:1822-6.

106. Eaton JK, Ruberto RA, Kramm A, Viswanathan VS, Schreiber SL. Diacylfuroxans are masked nitrile oxides that inhibit GPX4 covalently. J Am Chem Soc. 2019;141:20407-15.
107. Eaton JK, Furst L, Ruberto RA, Moosmayer D, Hilpmann A, Ryan MJ, et al. Selective covalent targeting of GPX4 using masked nitrile-oxide electrophiles. Nat Chem Biol. 2020;16:497-506.

108. Shimada K, Skouta R, Kaplan A, Yang WS, Hayano M, Dixon SJ, et al. Global survey of cell death mechanisms reveals metabolic regulation of ferroptosis. Nat Chem Biol. 2016:12:497-503.

109. Abrams RP, Carroll WL, Woerpel KA. Five-membered ring peroxide selectively initiates ferroptosis in cancer cells. ACS Chem Biol. 2016;11:1305-12.

110. Llabani E, Hicklin RW, Lee HY, Motika SE, Crawford LA, Weerapana E, et al Diverse compounds from pleuromutilin lead to a thioredoxin inhibitor and inducer of ferroptosis. Nat Chem. 2019;11:521-32.

111. Yang WS, Kim KJ, Gaschler MM, Patel M, Shchepinov MS, Stockwell BR. Peroxidation of polyunsaturated fatty acids by lipoxygenases drives ferroptosis. Proc Natl Acad Sci USA. 2016;113:E4966-75.

112. Gao J, Yang F, Che J, Han Y, Wang Y, Chen N, et al. Selenium-encoded isotopic signature targeted profiling. ACS Cent Sci. 2018;4:960-70.

113. Shah R, Shchepinov MS, Pratt DA. Resolving the role of lipoxygenases in the initiation and execution of ferroptosis. ACS Cent Sci. 2018;4:387-96.

114. Tonnus W, Meyer C, Steinebach C, Belavgeni A, von Massenhausen A, Gonzalez $\mathrm{NZ}$, et al. Dysfunction of the key ferroptosis-surveilling systems hypersensitizes mice to tubular necrosis during acute kidney injury. Nat Commun. 2021;12:4402.

115. Devisscher L, Van Coillie S, Hofmans S, Van Rompaey D, Goossens K, Meul E, et al. Discovery of novel, drug-like ferroptosis inhibitors with in vivo efficacy. J Med Chem. 2018;61:10126-40.

116. Hofmans S, Berghe TV, Devisscher L, Hassannia B, Lyssens S, Joossens J, et al. Novel ferroptosis inhibitors with improved potency and ADME properties. J Med Chem. 2016;59:2041-53.

117. Li C, Deng X, Zhang W, Xie X, Conrad M, Liu Y, et al. Novel allosteric activators for ferroptosis regulator glutathione peroxidase 4. J Med Chem. 2019;62:266-75.

118. Gao M, Monian P, Pan Q, Zhang W, Xiang J, Jiang X. Ferroptosis is an autophagic cell death process. Cell Res. 2016;26:1021-32.

119. Yan B, Ai Y, Sun Q, Ma Y, Cao Y, Wang J, et al. Membrane damage during ferroptosis is caused by oxidation of phospholipids catalyzed by the oxidoreductases POR and CYB5R1. Mol Cell. 2021;81:355-69. e10

120. Garcia-Bermudez J, Baudrier L, Bayraktar EC, Shen Y, La K, Guarecuco R, et al Squalene accumulation in cholesterol auxotrophic lymphomas prevents oxidative cell death. Nature. 2019;567:118-22.

121. Mancias JD, Wang X, Gygi SP, Harper JW, Kimmelman AC. Quantitative proteomics identifies NCOA4 as the cargo receptor mediating ferritinophagy. Nature. 2014;509:105-9.

122. Xu C, Xiao Z, Wang J, Lai H, Zhang T, Guan Z, et al. Discovery of a potent glutathione peroxidase 4 inhibitor as a selective ferroptosis inducer. J Med Chem. 2021;64:13312-26.

123. Gaschler MM, Andia AA, Liu H, Csuka JM, Hurlocker B, Vaiana CA, et al. FINO2 initiates ferroptosis through GPX4 inactivation and iron oxidation. Nat Chem Biol. 2018;14:507-15.

124. Rees MG, Seashore-Ludlow B, Cheah JH, Adams DJ, Price EV, Gill S, et al. Correlating chemical sensitivity and basal gene expression reveals mechanism of action. Nat Chem Biol. 2016;12:109-16.

125. Viswanathan VS, Ryan MJ, Dhruv HD, Gill S, Eichhoff OM, Seashore-Ludlow B, et al. Dependency of a therapy-resistant state of cancer cells on a lipid peroxidase pathway. Nature. 2017;547:453-7.

126. Ma S, Henson ES, Chen Y, Gibson SB. Ferroptosis is induced following siramesine and lapatinib treatment of breast cancer cells. Cell Death Dis. 2016;7:e2307.

127. Sun X, Ou Z, Chen R, Niu X, Chen D, Kang R, et al. Activation of the p62-Keap1NRF2 pathway protects against ferroptosis in hepatocellular carcinoma cells. Hepatology. 2016;63:173-84.

128. Hasegawa $M$, Takahashi $H$, Rajabi $H$, Alam M, Suzuki $Y$, Yin $L$, et al. Functional interactions of the cystine/glutamate antiporter, CD44v and MUC1-C oncoprotein in triple-negative breast cancer cells. Oncotarget. 2016;7:11756-69.

129. Sun X, Ou Z, Xie M, Kang R, Fan Y, Niu X, et al. HSPB1 as a novel regulator of ferroptotic cancer cell death. Oncogene. 2015;34:5617-25.

130. Fang $X$, Cai Z, Wang $H$, Han D, Cheng Q, Zhang $P$, et al. Loss of cardiac ferritin $H$ facilitates cardiomyopathy via Slc7a11-mediated ferroptosis. Circ Res. 2020;127:486-501

131. Yang WS, Stockwell BR. Synthetic lethal screening identifies compounds activating iron-dependent, nonapoptotic cell death in oncogenic-RAS-harboring cancer cells. Chem Biol. 2008;15:234-45.

132. Song X, Xie Y, Kang R, Hou W, Sun X, Epperly MW, et al. FANCD2 protects against bone marrow injury from ferroptosis. Biochem Biophys Res Commun. 2016:480:443-9.

133. Zhang Z, Zhang F, An P, Guo X, Shen Y, Tao Y, et al. Ferroportin1 deficiency in mouse macrophages impairs iron homeostasis and inflammatory responses. Blood. 2011;118:1912-22. 
134. Cao J, Chen X, Jiang L, Lu B, Yuan M, Zhu D, et al. DJ-1 suppresses ferroptosis through preserving the activity of S-adenosyl homocysteine hydrolase. Nat Commun. 2020;11:1251.

135. Eberhard $Y$, McDermott SP, Wang X, Gronda M, Venugopal A, Wood TE, et al. Chelation of intracellular iron with the antifungal agent ciclopirox olamine induces cell death in leukemia and myeloma cells. Blood. 2009;114:3064-73.

136. Yang W, Liu X, Song C, Ji S, Yang J, Liu Y, et al. Structure-activity relationship studies of phenothiazine derivatives as a new class of ferroptosis inhibitors together with the therapeutic effect in an ischemic stroke model. Eur J Med Chem. 2021;209:112842.

137. Liu Y, Wang W, Li Y, Xiao Y, Cheng J, Jia J. The 5-lipoxygenase inhibitor zileuton confers neuroprotection against glutamate oxidative damage by inhibiting ferroptosis. Biol Pharm Bull. 2015;38:1234-9.

138. Raefsky SM, Furman R, Milne G, Pollock E, Axelsen P, Mattson MP, et al. Deuterated polyunsaturated fatty acids reduce brain lipid peroxidation and hippocampal amyloid beta-peptide levels, without discernable behavioral effects in an APP/PS1 mutant transgenic mouse model of Alzheimer's disease. Neurobiol Aging. 2018;66:165-76.

139. Alim I, Caulfield JT, Chen Y, Swarup V, Geschwind DH, Ivanova E, et al. Selenium drives a transcriptional adaptive program to block ferroptosis and treat stroke. Cell. 2019;177:1262-79.

140. Tuo QZ, Masaldan S, Southon A, Mawal C, Ayton S, Bush Al, et al. Characterization of selenium compounds for anti-ferroptotic activity in neuronal cells and after cerebral ischemia-reperfusion injury. Neurotherapeutics. 2021. https://doi. org/10.1007/s13311-021-01111-9.

\section{ACKNOWLEDGEMENTS}

We thank Drs. Yilong Zou, Boyi Gan, Li Jiang, and Yingying Yu for helpful discussion, and we apologize to our colleagues whose valuable contributions were not cited in this manuscript due entirely to space limitations. This study was supported by research grants from the National Natural Science Foundation of China (31970689 to J.M. and 31930057 to F.W.), the National Key R\&D Program of China (2018YFA0507801 to J.M. and 2018YFA0507802 to F.W.).

\section{AUTHOR CONTRIBUTIONS}

$\mathrm{JM}, \mathrm{JC}, \mathrm{XL}, \mathrm{CG}$, and FW wrote the manuscript, and all authors approved the submitted version.

\section{COMPETING INTERESTS}

The authors declare no competing interests.

\section{ADDITIONAL INFORMATION}

Correspondence and requests for materials should be addressed to Junxia Min or Fudi Wang.

Reprints and permission information is available at http://www.nature.com/ reprints

Publisher's note Springer Nature remains neutral with regard to jurisdictional claims in published maps and institutional affiliations.

(i) Open Access This article is licensed under a Creative Commons adaptation, distribution and reproduction in any medium or format, as long as you give appropriate credit to the original author(s) and the source, provide a link to the Creative Commons license, and indicate if changes were made. The images or other third party material in this article are included in the article's Creative Commons license, unless indicated otherwise in a credit line to the material. If material is not included in the article's Creative Commons license and your intended use is not permitted by statutory regulation or exceeds the permitted use, you will need to obtain permission directly from the copyright holder. To view a copy of this license, visit http://creativecommons. org/licenses/by/4.0/.

(c) The Author(s) 2022 